\title{
CSCI Young Investigators Forum Abstracts
}

\author{
CSCI PROGRAM \\ PROGRAMME SCRC
}

Toronto

25 September, 2008 / le 25 Septembre, 2008

Clin Invest Med 2008; 31 (4S): 1-26.

\section{OPTIMIZING VITAMIN D LEVELS IN PATIENTS WITH MULTIPLE SCLEROSIS}

Ahluwalia, PS, Metz, LM, Hanley, DA, The Calgary MS Clinic Team.

Hotchkiss Brain Institute, University of Calgary.

Background: Emerging research suggests that vitamin D plays an important role beyond bone health, particularly in immune function and may be important in people with Multiple Sclerosis (MS). MS clinic physicians in Calgary, Alberta believe that patients should have at least minimally sufficient $(>80 \mathrm{nmol} / \mathrm{L})$ serum $25(\mathrm{OH}) \mathrm{D}$ levels to maintain adequate bone health. Involving patients in the assessment and management of their own vitamin D needs may be effective and more efficient than having clinicians track levels.

Objectives: Determine the prevalence of vitamin D insufficiency and the feasibility of using different management methods to optimize serum $25(\mathrm{OH}) \mathrm{D}$ levels.

Methods: 213 patients who attended the Calgary MS Clinic between September 2006 and January 2007 participated in this study. Each patient agreed to have serum $25(\mathrm{OH}) \mathrm{D}$ levels measured, and to adjust their vitamin D dose according to an algorithm that they would follow or that would be used by a graduate student to recommend dose changes at baseline, 3- and 6months.

Results: Mean age was 45.6 years (range 21-72); 78.9\% were women. Mean EDSS was 3.2 (range 0-8.5). Mean baseline serum level was $72.8 \mathrm{nmol} / \mathrm{L}$ (SD 26.8) (range 17.9-160.0); 62.4\% had levels $<80 \mathrm{nmol} / \mathrm{L}$. $60.6 \%$ of subjects were taking at least 1000 IU.

Conclusions: We found a high prevalence of vitamin D insufficiency despite a sizeable proportion taking at least $1000 \mathrm{IU}$ of vitamin D3 daily. Six month data, including adherence to the study protocol and proportion of participants with optimized $25(\mathrm{OH}) \mathrm{D}$ levels will be presented. Insight into the management of dosing for patients could have an impact on the integration of vitamin D optimization into the MS population.

\section{SOLUBLE PHOSPHOLIPASEA 2 IN THE LIFE AND DEATH OF OSTEOCLASTS}

\section{H. Allard-Chamard, P. Dufort, A. J. de Brum-Fernandes}

Division of Rheumatology, Faculté de médecine et des sciences de la santé, Université de Sherbrooke, Québec, Canada

Background: Prostaglandins are important for the control of bone remodeling; phospholipases $\mathrm{A}_{2}$ are essential for prostaglandin synthesis and could be an important mediator for bone health. Our objective was to determine the role of $\mathrm{SPLA}_{2}$ on osteoclastogenesis and osteoclast (OC) apoptosis.

Methods: Human osteoclasts were differentiated in vitro from PBMC in the presence of RANKL and M-CSF. The presence of sPLA $_{2}$ was confirmed by immunohistochemistry in OCs in culture and on bone slices. The role of sPLA2 II on osteoclastogenesis and on OC apoptosis was studied using sPLA2 inhibitors The implication of sPLA2 II in bone remodeling in vivo was determined by comparing levels of the enzyme in the peripheral blood of patients with traumatic fracture compared to age- and sexmatched controls.

Results: In vitro differentiated osteoclasts and in situ fetal and Pagetic bone osteoclasts express sPLA $_{2}$ II. sPLA 2 II inhibition during osteoclastogenesis reduces the capacity to generate osteoclasts and increases apoptosis rate of osteoclastic precursors; it also induces apoptosis on mature OCs. Patients with bone fractures have significantly less circulating $\mathrm{SPLA}_{2}$ II than controls.

Conclusion: $\mathrm{sPLA}_{2} \mathrm{II}$ is present in OCs both in vitro and in vivo, in normal as well as in pathologic situations. Its inhibition reduces osteoclastogenesis and increases apoptosis rate in vitro. Plasmatic sPLA 2 II levels are lower post-fracture than in controls. These results strongly suggest a role for sPLA2 in the control of bone metabolism but the mechanisms implicated in this effects still need to be clarified. 


\section{MANAGEMENT AND OUTCOME OF STAGE I SEMINOMA IN ONTARIO.}

\author{
Howard H An, Paul Y Peng, William J Mackillop. \\ Division of Cancer Care and Epidemiology, Queen's University \\ Cancer Research Institute, Kingston, Ontario
}

Purpose: To describe the uptake and use of surveillance and irradiation in stage I seminoma post-orchiectomy in Ontario and to evaluate the impact of these management strategies on patient outcomes.

Background: Testicular cancer is the most common cancer in men between the ages of 20 and 44 (1). About half of testicular cancers are seminomas (2). Overall $85 \%$ of seminoma patients present with stage I disease with 10-year survival over $99 \%$. Adjuvant radiation therapy once constituted the standard of care but surveillance as a post-orchiectomy management strategy is now preferred in Ontario (3). Treatment with radiation results in important long-term toxicities (2). The actual management patterns and the effect of these patterns on seminoma patient outcomes is unknown. This will be the first phase IV study to describe the management of stage I seminoma and to evaluate it's effect on patient outcomes.

Methods: This is a retrospective, population based cohort study of seminoma patients in Ontario. Cases of seminoma are identified through the Ontario Cancer Registry and linked with patient data from the Canadian Institute of Health Information and Ontario radiotherapy data. An instrumental variable approach will be taken with time and location of treatment as the instruments. Mantel-Haensel Chi-Squared tests, Student's T-test, and Log rank tests will be used to find differences in patient characteristics, morbidity and survival. The Kaplan-Meier method will be used to model overall survival.

Results and Conclusions: Pending data analysis.

\section{References:}

1. Warde P, Srugeon J, Gospodarowicz M. Testicular Cancer. In: Gunderson L, Tepper J, eds. Clinical radiation oncology. Philadelphia: Chruchill Livingstone; 2000: 844-862.

2. Nichols CR, Hung A, Corless CL, Foster RS, Roth BJ, Einhorn LH. Testis cancer. In: Kufe DW, Frei III E, Holland JF, Weichselbaum RR, Pollock RE, Bast Jr RC, Hong WK, Hait WN, eds. Holland-Frei cancer medicine $-7^{\text {th }}$ Ed.[e-book], Columbia: BC Decker; 2006 [cited 2008 Mar 17]: ch. 99. Available from: Stat!Ref.

3. Chung P, Mayhew LA, Warde P, Winquist E, Lukka H et al. Management of stage I seminoma: Guideline recommendations. Cancer Care Ontario, Evidence-Based Series \#3-18: Section 1; Report date: 30 Jan 2008.

\section{MOLECULAR CONTROL OF ARTICULAR CARTILAGE DEGENERATION BY TRANSFORMING GROWTH FACTOR ALPHA}

\section{Tom Appleton', Shirine Usmani', John Mort ${ }^{2}$, Frank Beier ${ }^{1}$}

${ }^{1}$ CIHR Group in Skeletal Development \& Remodeling, Dept. of Physiology \& Pharmacology, University of Western Ontario, London, ON, Canada; ${ }^{2}$ Shriner's Hospital, McGill University, Montreal, QC, Canada

Background: Articular cartilage degeneration is a hallmark of osteoarthritis (OA). We previously identified increased expression of transforming growth factor alpha (TGF $\alpha$ ) and chemokine (C-C motif) ligand 2 (CCL2) in articular cartilage from a rat model of OA $(1,2)$. We subsequently reported that TGF $\alpha$ signalling modified chondrocyte cytoskeletal organization, increased catabolic and decreased anabolic gene expression and suppressed Sox9. Due to other roles in chondrocytes, we hypothesized that the effects of TGF $\alpha$ on chondrocytes are mediated by Rho/ROCK and MEK/ERK signaling pathways.

Methods: Primary cultures of chondrocytes and articular osteochondral explants were treated with pharmacological inhibitors of MEK1/2 (U0126), ROCK (Y27632), Rho (C3), p38 MAPK (SB202190) and PI3K (LY294002) to elucidate pathway involvement.

Results: Using G-LISA we determined that stimulation of primary chondrocytes with TGF $\alpha$ activates RhoA. Reciprocally, inhibition of RhoA/ROCK but not other signalling pathways prevents modification of the actin cytoskeleton in response to TGF $\alpha$. Inhibition of MEK/ERK signaling rescued suppression of anabolic gene expression by TGF $\alpha$ including SOX9 mRNA and protein levels. Inhibition of MEK/ERK, Rho/ROCK, p38 MAPK and PI3K signalling pathways differentially controlled the induction of MMP13 and TNF $\alpha$ gene expression. TGF $\alpha$ also induced expression of CCL2 specifically through MEK/ERK activation. In turn, CCL2 treatment induced the expression of MMP3 and TNF $\alpha$. Finally, we assessed cartilage degradation by immunohistochemical detection of type II collagen cleavage fragments generated by MMPs. Blockade of RhoA/ROCK and MEK/ERK signalling pathways reduced the generation of type II collagen cleavage fragments in response to TGF $\alpha$ stimulation.

Conclusions: Rho/ROCK signalling mediates TGF $\alpha$-induced changes in chondrocyte morphology, while MEK/ERK signalling mediates the suppression of Sox9 and its target genes, and CCL2 expression. CCL2, in turn, induces the expression of MMP3 and TNF $\alpha$, two potent catabolic factors known to be involved in OA. These pathways may represent strategic targets for interventional approaches to treating cartilage degeneration in osteoarthritis.

\section{References:}

1. Appleton CTG et al. Arthritis Rheum 2007;56:1854-68.

2. Appleton CTG et al. Arthritis Rheum 2007;56:3693-705. 


\section{THE TRANSCRIPTION FACTOR Y-BOX BINDING PROTEIN-1 (YB-1) INDUCES EXPRESSION OF THE PIK3CA ONCOGENE LEADING TO INCREASED INVASION OF BASAL-LIKE BREAST CARCINOMA CELLS}

Arezoo Astanehe ${ }^{1,2}$, Melanie Finkbeiner ${ }^{2}$, Karen To ${ }^{2}$, Sandra E. Dunn ${ }^{2}$

${ }^{1} \mathrm{MD} / \mathrm{PhD}$ Program, Department of Experimental Medicine; ${ }^{2} \mathrm{La}-$ boratory for Oncogenomic

Research, Department of Paediatrics, University of British Columbia, Vancouver, BC.

Background: Basal-like breast carcinoma (BLBC) is the most aggressive subtype of breast cancer. $73 \%$ of BLBC over-express YB-1, an oncogenic transcription/translation factor. $P I K 3 C A$, which codes for the p $110 \alpha$ catalytic subunit of phosphatidylinositol-3-kinase (PI3K), is another oncogene. The PI3K signalling pathway is fundamental in the regulation of many cellular functions and is often deregulated in cancer. Despite its importance, the knowledge on the transcriptional regulation of $P I K 3 C A$ is limited. Indeed, we have recently published the first report on the $P I K 3 C A$ promoter.

Methods and Results: A genome-wide chromatin immunoprecipitation on chip (ChIP-on-chip) analysis of a BLBC cell-line (SUM149) suggested binding of YB-1 to the PIK3CA promoter. This binding was verified using traditional chromatin immunoprecipitation (ChIP). Furthermore, electrophoretic mobility shift assay (EMSA) using oligonucleotides with either wild-type or mutated YB-1 responsive elements mapped YB-1 binding to three sites on the PIK3CA promoter. Silencing YB-1 in BLBC cell-lines (SUM149, HCC1937, and MDA-MB-231) decreased, while overexpression of YB-1 increased the PIK3CA promoter activity, transcript, and protein levels. Interestingly, array comparative genomic hybridization (aCGH) and quantitative PCR demonstrated PIK3CA copy number gains in HCC1937 and MDA-MB-231 cells. Although $P I K 3 C A$ amplifications are overall uncommon $(9 \%)$ in breast cancer, we demonstrated here that low level gains in PIK3CA copy number are present in $30 \%$ of primary BLBC cases. Furthermore, it has previously been demonstrated that mutations of PIK3CA are the most common genetic aberration (27\%) found in breast cancer. These mutations lead to constitutive activation of $\mathrm{p} 110 \alpha$ and are highly oncogenic. Over-expression of YB-1 in MCF-7 cells, which harbour an activating PIK3CA mutation, increased $P I K 3 C A$ transcript and protein levels. Furthermore, induction of $P I K 3 C A$ by YB-1 leads to increased levels of urokinase plasminogen activator (uPA) and invasion.

Conclusions: Our data demonstrates that YB-1 binds to the $P I K 3 C A$ promoter and induces its expression whether the gene is wild-type or amplified. Moreover, since YB-1 induces expression of the active mutant $\mathrm{p} 110 \alpha$, then therapeutic inhibition of YB-1 may lead to decreased $\mathrm{p} 110 \alpha$ and interference with the constitutively activated PI3K pathway in cancers. In addition, the YB-1/ $P I K 3 C A / \mathrm{uPA}$ network provides information regarding the possible therapeutic targets for prevention of breast cancer invasion and metastasis.

A.A. is supported by a Child and Family-CIHR-UBC $\mathrm{MD} / \mathrm{PhD}$ Studentship Award.

\section{XMODULATION IN MICE AND MEN: IL-10 PRODUCING CELLS IN BLOOD AND LYMPHOID TISSUE.}

\section{Barrett ${ }^{1,2}$, M Grant ${ }^{1}$, R Liwski ${ }^{3}, K$ West $^{2}$.}

${ }^{1}$ Division of BioMedical Sciences, Faculty of Medicine, Memorial University of Newfoundland, St. John's, NL

${ }^{2}$ Department of Medicine, Dalhousie University, Halifax, NS ${ }^{3}$ Department of Pathology, Dalhousie University, Halifax, NS

Background: The human immune system provides remarkable protection from a plethora of pathogens, but can cause damage when activated for a prolonged time (as in persistent infections) or against self (autoimmunity). Therefore, mechanisms of immune system downregulation and control are imperative. There is little data on how the immune system is controlled in healthy individuals. We recently described a novel population of white blood cells that constitutively produce the immunomodulatory cytokine interleukin-10 (IL-10). Our objective was to further delineate the distribution of these cells in human and mouse models, as well as potential triggers for interleukin-10 production in vitro.

Methods: Human and animal protocols were reviewed and approved by the institutional ethics board and animal care facilities, and informed consent was obtained from all human donors. The ex vivo percentage of peripheral blood $\mathrm{CD} 36^{+} \mathrm{IL}-10^{+}$mononuclear cells was assessed by intracellular flow cytometry in 10 healthy individuals. IL-10 production after exposure to two CD36 ligands, thrombospondin and oxidized low density lipoprotein (oxLDL) was measured at 8 hours. Peripheral blood mononuclear cells and splenocytes from $\mathrm{BL} / 6(\mathrm{n}=5)$ and $\mathrm{Balb} / \mathrm{c}$ $(n=1)$ mice were assessed for $\mathrm{CD} 36^{+} \mathrm{IL}-10^{+}$cells ex vivo as well.

Results: The percentage of $\mathrm{CD} 36^{+} \mathrm{IL}-10^{+}$cells in peripheral blood from healthy individuals ranges between $0.1 \%$ and $0.9 \%$. The percentage was similar in mouse peripheral blood, with a range of $0.4 \%-1.1 \%$. These cells were also found in mouse spleen at a higher frequency than peripherally $(1.1-1.5 \%)$. Human $\mathrm{CD} 36^{+} \mathrm{IL}-10^{+}$cells have more IL-10 when exposed to thrombospondin, oxLDL.

Conclusions: Our novel population of IL-10 producing cells is found not only in healthy humans, but also in lymphoid tissue and blood from pathogen free mice. This highlights the evolutionary conservation of the cell across species, and suggests an important homeostatic function. The physiologic ligands for CD36 are ubiquitous in circulation, and our in vitro data suggests a link between CD36 ligation and IL-10 production. IL-10 is a known immune system modulator, and its production by these cells may help maintain homeostatic control of the immune system. 


\section{SELECTIVE USE OF MEMBRANE CD14, BUT NOT SOLUBLE CD14 AND INHIBITION BY SERUM IN THE INFLAMMATORY CYTOKINE RESPONSE INDUCED BY A NOVEL TLR LIGAND (P. AERUGINOSA EXOENZYME S)}

\author{
Berenger $\mathrm{B}^{1}$, Mody $\mathrm{CH}^{2}$ \\ Departments of ${ }^{1}$ Medical Science and ${ }^{2}$ Internal Medicine and \\ Microbiology and Infectious Diseases. Faculty of Medicine, \\ University of Calgary. 3300 Hospital Drive NW T2N 1N4. Cal- \\ gary, Alberta, Canada.
}

Background/Purpose: Soluble and membrane CD14 (sCD14 and $\mathrm{mCD} 14)$ bind microbial products, are toll-like receptor (TLR) coreceptors, and induce inflammation. Clinical relevance is demonstrated by a correlation of sCD14 levels with morbidity and mortality in inflammatory diseases. Moreover, $P$. aeruginosa induces damaging inflammation in the lungs of cystic fibrosis patients and its virulence is often attributed to the TLR2 and TLR4 ligand exoenzyme S (ExoS). Because of the importance of the response of CD14 to TLR ligands and its potential for therapeutic manipulation, its role in ExoS-induced inflammatory cytokines was investigated.

Methods: The ability of ExoS to induce TNF and IL-6 cytokine production in cell lines that express TLR and either CD14 positive or negative was assessed (THP-1 or U373 cells, respectively). Recombinant CD14 or CD14 blocking agents were used to test the contribution of mCD14 and sCD14.

Results: Enhancing expression of mCD14 on THP-1 cells increased TNF production, which was abrogated by blocking or removing mCD14. Transfecting mCD14 into U373 cells demonstrated that mCD14 was required for binding ExoS and subsequent IL-6 production. Unlike TLR ligands that only stimulate one TLR, such as lipopolysaccharide, neither sCD14 nor serum enhanced production of cytokine in response to ExoS. Uniquely, serum inhibited ExoS induced TNF.

Conclusion: This work demonstrates a fundamental difference in the requirement of ExoS and other TLR ligands for CD14, which must be considered when designing therapies to block microbeinduced inflammation. The presence of a potential therapeutic molecule in serum could help in the development of an ExoS neutralizing agent.

\section{THE IMPACT OF ESTIMATED GLOMERULAR FILTRATION RATE REPORTING ON NEPHROLOGY REFERRAL PATTERN, PATIENT CHARACTERISTICS AND OUTCOME}

\section{Arik Bergman, David MJ Naimark}

University of Toronto CIP program

Background: Chronic kidney disease (CKD) is a growing public health problem worldwide. The key to reducing the burden of CKD is early detection and delay of disease progression. An elevated serum creatinine concentration, $[\mathrm{Cr}]$, is a common indicator of CKD. However, even with advanced $\mathrm{CKD},[\mathrm{Cr}]$ may not be high among patients with low muscle mass (particularly the elderly). Thus, the use of the estimated glomerular filtration rate (eGFR) has been advocated for identifying severe occult kidney disease. In January 2006, community laboratories in Ontario, Canada, began to report an eGFR value along with every [Cr] result. The present study sought to investigate the impact of eGFR reporting on nephrology referrals and subsequent patient outcome.

Methods: The current study consisted of a retrospective analysis of all referrals to the adult general nephrology clinics at Sunnybrook Health Sciences Centre 15 months before and after eGFR reporting took effect on January 1, 2006.

Results: eGFR reporting was associated with a significant rise in the number of referrals ( 971 to $1101, \mathrm{p}=0.03$ ), a $51 \%$ rise in patient wait-time (from 73 days to 110 days, $\mathrm{p}<0.001$ ) and an increase in nephrologists' workload. Patients referred after eGFR reporting were older, but suffered from fewer comorbidities such as hypertension, vascular disease, and dementia. There was an increase in the number of patients referred with stage $3 \mathrm{CKD}$, but no change in the proportion of stage 4 and $5 \mathrm{CKD}$ referrals or the number of patients initiating dialysis.

Conclusion: Laboratory reporting of eGFR increased nephrology referral volume, patient waiting times, and nephrologists' workload, without a demonstrable benefit in terms of detection and referral of severe (stage 4 and 5) CKD nor in the reduction of ESRD frequency. Screening programs may need to be implemented along with knowledge translation strategies in order to achieve the goal of preventing and delaying progression of CKD.

Reference: Canadian Society of Nephrology, September 2006. Care and referral of adult patients with reduced kidney functionPosition paper from the Canadian Society of Nephrology. Available at:

http://www.csnscn.ca/local/files/CSN-Documents/CSN\%20Posti on $\% 20$ Paper $\% 20$ Sept $2006 . p d f$ 
"IT'S MORE THAN A PILL DROP": ADHERENCE SUPPORT AND THE CLINICAL GAZE IN INNER CITY HOME-BASED HIV CARE

\author{
S. Berkhout ${ }^{1}$, E. Brennan ${ }^{2}$, S. Giles ${ }^{2}$, B. Joyce $^{2}$, \\ S. Smyth ${ }^{2}$, M. Tyndall \\ ${ }^{1} \mathrm{MD} / \mathrm{PhD}$ Program, Vancouver, Canada, University of British \\ Columbia, Vancouver, BC, \\ ${ }^{2}$ Vancouver Coastal Health, Vancouver, BC, \\ ${ }^{3} \mathrm{BC}$ Centre for Excellence in HIV/AIDS, Epidemiology, Van- \\ couver, $\mathrm{BC}$
}

Background: Home-based HIV care has been identified as means of improving access to HIV care and reducing HIV-related stigma, while easing overload in facility-based care and providing cost effective community-based social supports. Few evaluations, however, focus on the impact of barriers such as homelessness, imprisonment and substance abuse to the continuity of this model of health care provision. This paper examines the challenges, strategies, and outcomes of a long-standing home-based HIV nursing program from a Foucauldian analytic perspective.

Methods: Findings are presented from the evaluation of a home care nursing team providing HIV care to unstably housed individuals in Vancouver's inner city. Employing ethnographic methodology, participant observation was conducted over nine months, along with serial open-ended qualitative interviews with the nursing team $(n=4)$ and home care clients $(n=16)$. Interviews were audio recorded and transcribed; an interpretive thematic analysis of field notes and interview transcripts was conducted.

Results: Although adherence to antiretroviral therapy was consistent over the study course, men and women's responses to care varied over time. Nurses who were sensitive to the ways in which enhanced surveillance within the private sphere of participants' lives was cause for resistance to care employed a number of strategies to maintain these relationships. Variability among nurses regarding local (i.e. street) knowledge, along with understandings of what constitutes appropriate supportive measures, also impacted participants' responses to care.

Conclusions: The type and extent of support services is continually negotiated between nurses and participants, creating a potential source of tension between the guiding of health-related decisions and participants' constitution of themselves as autonomous. Foucault's concept of governmentality is applicable here, and may elucidate how norms, capacities and subjectivities are mutually created through its processes. The findings support an understanding of autonomy that is interpersonally, as well as institutionally, relational. Successful programming requires careful attention to the everyday experiences of participants, facilitated by continuity of providers and mentorship for newer nurses engaging in home-based HIV care.

Suze Berkhout is supported by the Canadian Institutes for Health Research, The Michael Smith Foundation for Health Research, Providence Health, and Vancouver Coastal Health Research Institute.

\section{RELATIVE CONTRIBUTIONS OF FIBROSIS AND CONNEXIN CHANGES TO THE ATRIAL FIBRILLATION SUBSTRATE DURING THE DEVELOPMENT AND REVERSAL OF CONGESTIVE HEART FAILURE}

Brett Burstein $^{1,2,3}$, Kunihiro Nishida ${ }^{2}$, Philippe Comtois ${ }^{3}$, Louis Villenuve $^{3}$, Yung-Hsin Yeh ${ }^{3}$, Tack-Ki Leung ${ }^{3}$, Stanley Nattel ${ }^{2.3}$

${ }^{1} \mathrm{MD} / \mathrm{PhD}$ Program, McGill University, Montreal, QC, ${ }^{2}$ Department of Pharmacology and Therapeutics, McGill University, Montreal, QC, ${ }^{3}$ Research Centre, Montreal Heart Institute, Montreal, QC.

Background: Connexin alterations occur in various atrial fibrillation (AF) paradigms, but their functional significance remains unclear. No data are available regarding the effects of $\mathrm{CHF}$ on atrial connexin expression and phosphorylation. We therefore analyzed connexin changes and their contribution to the AF substrate during the development and reversal of CHF.

Methods and Results: Dogs were allocated to three groups: $\mathrm{CHF}$ induced by 2-week ventricular tachypacing $(\mathrm{CHF}, \mathrm{n}=15)$; CHF dogs allowed to recover for 4 weeks after 2-week tachypacing (REC, $n=15)$ and non-paced shams (CTL, n=11). Left ventricular end-diastolic pressure increased with $\mathrm{CHF}$ $(14.5 \pm 1.0 * * *$ vs. $3.7 \pm 0.7, * * * P<0.001$ vs. CTL) and normalized upon CHF recovery $\left(5.1 \pm 1.0^{\dagger \dagger \dagger}, \dagger^{\dagger \dagger} P<0.001\right.$ vs. CHF). Real-time PCR and Western-blot analyses revealed connexin43 (Cx43) and connexin40 (Cx40) mRNA and protein expression to be unchanged by $\mathrm{CHF}$ and REC. However, CHF caused Cx43 dephosphorylation (by $\sim 73 \% * * *$ ) and increased $\mathrm{Cx} 40 / \mathrm{Cx} 43$ protein ratio (by $\sim 35 \% * * *$ ), with both alterations completely reversing in REC. Immunofluorescent confocal microscopy confirmed connexin protein trends, with a reduction in phosphorylated $\mathrm{Cx} 43$ (by $\sim 68 \% * * *$ in $\mathrm{CHF}$ ) that returned to control in REC. $\mathrm{CHF}$ caused conduction abnormalities (phase delay-range and heterogeneity index, both $P<0.01$ ) and burst pacing-induced AF

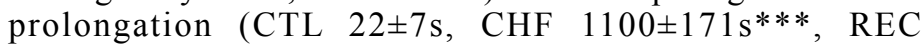
$884 \pm 220 \mathrm{~s}^{* * *}$ ) which persisted in the recovery period, along with residual fibrosis (CTL 3.6 $\pm 0.7 \%$, CHF $14.7 \pm 1.5 \% * * *, \mathrm{REC}$ $13.3 \pm 2.3 \% * * *)$. Fibrosis physically interrupted muscle bundle continuity and an ionically-based action potential model of canine atrium showed that fibrosis was able to account for the observed conduction abnormalities.

Conclusions: $\mathrm{CHF}$ causes connexin-dephosphorylation and $\mathrm{Cx} 40 / \mathrm{Cx} 43$ ratio increases. With $\mathrm{CHF}$ reversal, atrial connexin alterations recover completely, but tissue fibrosis, conduction abnormalities and a substrate for AF remain with fibrosis accounting for conduction abnormalities. Thus, although atrial connexin changes occur with CHF, they are not essential for conduction disturbances and AF promotion, which appear rather to be related primarily to fibrotic interruption of muscle-bundle continuity. 


\section{CONCEPTION AND VALIDATION OF A REFLUX LARYNGITIS MODEL IN NEWBORN LAMBS}

\section{A-M Carreau, H Patural, A Doueik, P-H Fortier, JP Praud}

Surgery and pediatrics department, Université de Sherbrooke.

Background: Daily clinical observations and a recent study (D Vermeylen et al., 2004) suggest that reflux laryngitis (RL) is involved in apneas/ bradycardias of the newborn. The aim of this study is to develop and to validate a unique RL model in newborn lambs to test the assumption that a RL provokes an increase in apneas/ bradycardias/ desaturations observed within the laryngeal chemoreflexes induced by the contact of a liquid with the larynx.

Methods: A surgical instrumentation was performed on the first day of life, which included the insertion of a transcutaneous esophageal catheter in order to allow instillation of a gastric juice surrogate $(\mathrm{HCl}+$ pepsin at $\mathrm{pH} 2)$ directly on the larynx. The 2 ml-instillations were made 3 times per day for 6 consecutive days. At day 9-10 laryngeal chemoreflexes were induced during sleep. The larynx was removed at the time of necropsy to grade inflammation by a histological study.

Results: Lambs in which a RL was induced $(n=4)$ presented characteristic signs of RL: cough, weak bleat, reduction in weight gain. Preliminary histological analyses showed a characteristic laryngeal inflammation. Preliminary results showing that apneas and oxygen desaturations seem more prominent in RL lambs than controls provided further validation of our RL model.

Conclusion: The ovine model developed in this study presents the clinical and histological characteristics of RL observed in newborns. Preliminary results suggesting that a RL increases apneas/bradycardias/desaturations when a liquid is in contact with laryngeal mucosa in the newborn must be confirmed.

Supported by: CIHR, FRSQ, Foundation of stars.

\section{INVESTIGATING THE ROLE AND REGULATION OF THE SPECIFIC AND GENERAL PRB-E2F1 INTERACTIONS}

\author{
Matthew J. Cecchini ${ }^{1,2,}$ \& Frederick A. Dick ${ }^{1,2}$ \\ ${ }^{1}$ London Regional Cancer Program, ${ }^{2}$ Department of Biochemis- \\ try, Schulich School of Medicine, University of Western Ontario, \\ London, Ontario
}

Background/Purpose. The retinoblastoma tumor suppressor protein (pRB) plays a central role in proliferative control and is a frequent target for inactivation in cancer. The G1-S phase transition of the cell cycle is regulated by pRB, which is capable of interacting with E2F family members and inhibiting the transcription of genes required to progress into S-phase. E2F1 is unique from other E2F family members as it can induce both apoptosis and proliferation. To control these contrasting functions of $\mathrm{E} 2 \mathrm{~F} 1$, a second $\mathrm{E} 2 \mathrm{~F} 1$ binding site exists in the Cterminus of $\mathrm{pRB}$ that can control apoptosis separately from proliferation. This anti-apoptotic function of $\mathrm{pRB}$ can in some circumstances promote tumorigenesis, which leads to the question; is $\mathrm{pRB}$ a tumor suppressor or an oncogene?

Methods. To investigate this, a gene-targeted mouse model is being engineered to selectively disrupt the ability of pRB to control proliferation through the general E2F binding site while still retaining the ability to control apoptosis through the specific E2F1 site.

Results. A series of novel mutants were engineered to selectively disrupt the binding of E2Fs at the general site, and prevent $\mathrm{pRB}$ from controlling proliferation. The mutants retain the ability to bind E2F1 and control apoptosis through the specific binding site, which is not disrupted.

Conclusions. By separating the ability of pRB to control proliferation and apoptosis under in vivo conditions, a better understanding into the significance of these two functions in development and tumorigenesis can be gained. 


\section{DISSECTING THE SIGNALLING PATHWAYS INVOLVED IN THE ANTI-HYPERTROPHIC EFFECTS OF RESVERATROL}

\author{
Anita Y.M. Chan, Vernon W. Dolinsky, Carrie-Lynn M. Soltys, \\ and Jason R.B. Dyck
}

Depts of Pediatrics and Pharmacology, Faculty of Medicine and Dentistry, University of Alberta

Background: Pathological left ventricular hypertrophy is associated with all-cause mortality; however, effective treatment for this condition is currently lacking. We have shown that activation of AMP-activated protein kinase (AMPK) by resveratrol can inhibit myocardial hypertrophy by decreasing protein synthesis and suppressing nuclear factor of activated T-cells (NFAT) activation. However, the mechanism by which resveratrol affects AMPK is unknown. Since LKB1 is the upstream kinase of AMPK, we hypothesize that resveratrol signals via LKB1 to activate AMPK and it is this signalling pathway that contributes to the anti-hypertrophic effects of resveratrol.

Methods: Wildtype (WT), LKB1 null, and AMPK null mouse embryonic fibroblasts (MEFs) were treated with vehicle or $100 \mu \mathrm{M}$ resveratrol for $1 \mathrm{~h}$. Cell lysates were subjected to immunoblot analysis to examine the phosphorylation status of the proteins of interest. NFAT-dependent transcription was also measured in these MEFs using a NFAT-luciferase reporter transgene.

Results: While resveratrol treatment increased AMPK phosphorylation in WT MEFs, resveratrol was unable to activate AMPK in LKB1 null MEFs. In addition, resveratrol suppressed NFATdependent transcription in WT MEFs, yet failed to inhibit NFAT activity in AMPK null MEFs.

Conclusion: These data combined with our previous data suggest that resveratrol signals through LKB1 to activate AMPK and that this activation results in suppressed protein synthesis and reduced NFAT activation. As the development of pathological cardiac hypertrophy is dependent on protein synthesis and NFAT activation, inhibition of these two pathways by resveratrol may be an exciting new approach for the treatment of pathological cardiac hypertrophy.

\section{CHEMICAL GENETICS REVEALS A COMPLEX FUNCTIONAL GROUND STATE OF NEURAL STEM CELLS}

Phedias Diamandis ${ }^{1-4}$, Jan Wildenhain ${ }^{4}$, Ian D Clarke ${ }^{1,2}$, Adrian G Sacher 1,2, Jeremy Graham ${ }^{1,2}$, David S Bellows ${ }^{3}$, Erick K M Ling ${ }^{1,2,5}$, Ryan J Ward ${ }^{1,2,5}$, Leanne G Jamieson ${ }^{1,2,5}$, Mike Tyers $^{3,4}$, Peter B Dirks ${ }^{1,2,5,6}$

${ }^{1}$ Arthur and Sonia Labatt Brain Tumor Research Ctr and

${ }^{2}$ Program in Developmental \& Stem Cell Biology, Hospital for Sick Children and Univ. of Toronto,

${ }^{3}$ Samuel Lunenfeld Research Inst., Mount Sinai Hospital, ${ }^{4}$ Dept of Medical Genetics \& Microbiology, Univ. of Toronto, ${ }^{5}$ Dept of Laboratory Medicine \& Pathobiology, Univ. of Toronto, ${ }^{6}$ Neurosurgery Div., Hospital for Sick Children \& Univ. of Toronto

The identification of self-renewing and multipotent neural stem cells (NSCs) in the mammalian brain holds promise for the treatment of neurological diseases and has yielded new insight into brain cancer. However, the complete repertoire of signaling pathways that governs the proliferation and self-renewal of NSCs, which we refer to as the 'ground state', remains largely uncharacterized. Although the candidate gene approach has uncovered vital pathways in NSC biology, so far only a few highly studied pathways have been investigated. Based on the intimate relationship between NSC self-renewal and neurosphere proliferation8, we undertook a chemical genetic screen for inhibitors of neurosphere proliferation in order to probe the operational circuitry of the NSC. The screen recovered small molecules known to affect neurotransmission pathways previously thought to operate primarily in the mature central nervous system; these compounds also had potent inhibitory effects on cultures enriched for brain cancer stem cells. These results suggest that clinically approved neuromodulators may remodel the mature central nervous system and find application in the treatment of brain cancer.

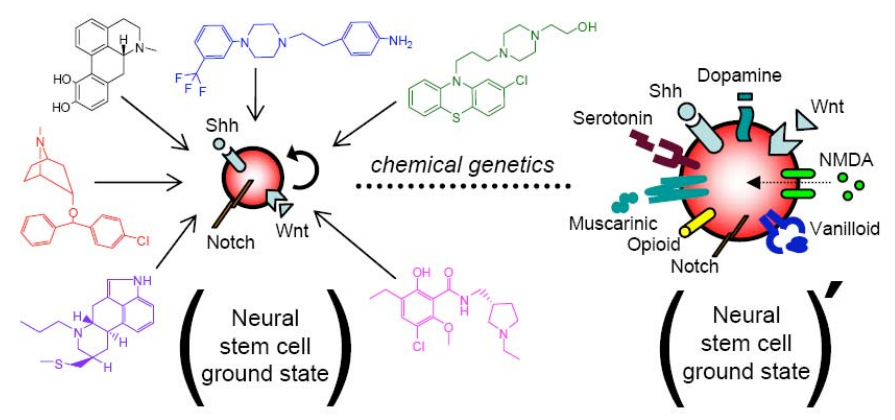




\section{GENERATION OF AN EFFECTIVE IN VIVO CTL RESPONSE USING VACCINE APPROACHES IN A MODEL OF AUTOIMMUNITY}

\author{
Dissanayake DT $T^{1}$ \& Ohashi PS ${ }^{2}$ \\ ${ }^{1}$ Department of Immunology, University of Toronto \\ ${ }^{2}$ Campbell Family Institute for Breast Cancer Research, Toronto
}

Background: Various interactions between tumours and their environment make it difficult to determine the factors that are essential to the induction of a robust CTL response by immunotherapeutic strategies against tissue antigens. We therefore sought to develop a model of autoimmunity, in which vaccine strategies could be evaluated for their ability to elicit CTL activity towards a model antigen.

Methods: Transgenic mice expressing lymphocytic choriomeningitis virus glycoprotein (LCMV-gp) specifically in the insulinproducing beta-islets of the pancreas allowed anti-beta islet CTL responses to be measured by blood glucose levels, tetramer and intracellular cytokine staining. Peptide vaccines consisted of i.v. administration of the known immunodominant epitopes of LCMV-gp alongside dendritic cell (DC) maturation stimuli. DC vaccines consisted of i.v. administration of $2 \times 10^{6}$ mature LCMVgp peptide-pulsed bone-marrow-derived DC.

Results: While administration of antigenic peptides with adjuvants and/or costimulatory molecules were ineffective, DC vaccination induced hyperglycemia in approximately $70 \%$ of mice. Interestingly, while a class II epitope was not required for induction of autoimmunity, pulsing with a single class I epitope was insufficient. Furthermore, while tetramer-positive populations could be seen in the blood of peptide-vaccinated mice, no such population is seen in DC-treated mice.

Conclusions: The lack of correlation between presence of tetramer-positive populations in the blood and effective beta-islet cell cytolysis underlines the requirement for better markers of CTL activity in clinical trials. These results demonstrate the effectiveness of DC vaccination in this model and allow for further investigation of the factors that direct a strong CD8+ T-cell response towards tissue antigens.

\section{THE P53 FAMILY MEMBER, P63, REGULATES NEURAL PRECURSOR CELL SURVIVAL DURING CORTICAL DEVELOPMENT}

\author{
Sagar Dugani, Annie Paquin, Masashi Fujitani, David R. \\ Kaplan, and Freda D. Miller \\ Departments of Developmental and Stem Cell Biology \& Cell \\ Biology, The Hospital for Sick Children; Institute of Medical \\ Science, Medical Sciences Building, University of Toronto
}

Background: p63, a member of the p53 family of proteins, is involved in the regulation of naturally-occurring apoptosis in sympathetic neurons of the peripheral nervous system. Since data from our laboratory indicated that p63 is also expressed in stem cells and neurons within the developing brain, we hypothesized that p63 is involved in regulating the genesis and survival of developing neurons.

Methods: As cortical neurogenesis is initiated at embryonic day 12 , we knocked-down p63 levels in isolated murine cortical precursors by using shRNA against p63 or by transfecting floxedp63 precursors with Cre recombinase. We performed similar studies in vivo using in utero electroporation to express either p63 shRNA or Cre recombinase to acutely knockdown or genetically ablate p63. We then performed immunofluorescence for known markers of apoptosis, cell-division, and differentiation to assess the level of cell death, proliferation and neurogenesis.

Results: Knock-down of p63 in vitro resulted in a 2-fold increase in the death of precursors and neurons, associated with blunted neurogenesis but unaltered precursor proliferation. Coincident knock-down of p63 family members, p53, but not p73, rescued the elevated death suggesting that p63 and p53 antagonize each other to promote survival. Similar results were observed in vivo, where knockdown of p63 caused cell death and a decrease in the proportion of neurons in the cortical plate.

Conclusions: These experiments indicate that p63 is required for the survival of neural precursors and newly-born neurons, and for normal cortical development. Ongoing work will explore the environmental cues that regulate p63 during neurogenesis. 


\section{OPPOSING FUNCTIONS FOR A PROTEIN KINASE: A JNK1 DEPENDENT SWITCH DETERMINES THE ONCOGENIC OR TUMOR SUPPRESSIVE ACTIVITY OF ILK IN RHABDOMYOSARCOMA}

Adam D. Durbin ${ }^{1,2,4}$, Gino R. Somers ${ }^{3,5}$, Michael Forrester 4 , Gregory E. Hannigan ${ }^{6}$, David Malkin 1,4 .

Department of Medical Biophysics ${ }^{1}, \mathrm{MD} / \mathrm{PhD}$ Department $^{2}$, Department of Laboratory Medicine and Pathobiology ${ }^{3}$, University of Toronto. Division of Hematology/Oncology-Departments of Pediatrics $^{4}$, Pediatric Laboratory Medicine ${ }^{5}$, The Hospital for Sick Children, Toronto, ON. Monash Institute of Medical Research $^{6}$, Monash University, Melbourne, Victoria, Australia.

Background: The integrin-linked kinase (ILK) is a protein kinase involved in the regulation of pathogenic cancer cell behaviours, such as proliferation, survival and invasion. ILK appears to be pro-oncogenic in vitro and in vivo models of tumorigenesis. Rhabdomyosarcoma (RMS) is a primitive mesenchyme-derived tumor and is subclassified into primarily embryonal (ERMS) and alveolar (ARMS) variants. Patients who present with metastatic RMS tumors have a less than $20 \%$ chance of cure, suggesting a need to define novel targets for chemotherapeutic intervention.

Methods: We used cell culture, murine xenografts and primary human tumors to examine ILK expression and functionality. RNAi and adenoviruses were used to knock down or overexpress proteins, and SP600125 was used to inhibit JNK kinase activity. ERMS cells stably expressing $P A X 3-F O X O 1 A$ were generated using pcDNA3.1 with the full length $P A X 3-F O X O 1 A$ cDNA insert.

Results: RNAi-mediated ablation of ILK induced stimulation of ERMS and inhibition of ARMS cell growth in vitro and in vivo. Overexpression of ILK, but not the ILK-R211A mutant reversed these effects. High-throughput screening of multiple tumor cell lines and mesenchymal progenitor cells demonstrated similar ILK anti-growth effects. Consistent with these results, clinical correlations made between ILK immunohistochemical staining intensity and patterns on an ERMS tumor tissue microarray revealed downregulation of ILK in stage III/IV primary tumors. Mechanistically, ILK silencing induced selective phosphorylation of the c-jun amino terminal kinase (JNK) and its target c-Jun in ERMS cells with attenuated phosphorylation in ARMS cells. ERMS cells express higher levels of JNK1 isoforms than ARMS cells. Introduction of the ARMS-associated $P A X 3-F O X O 1 A$ fusion gene into ERMS cells restored the oncogenic function of ILK and downregulated of JNK1. Coupling ILK siRNA with inhibition of the JNK-c-Jun signaling pathway in ERMS cells resulted in growth reductions and apoptotic induction. In contrast, coupling ILK knockdown with overexpression of JNK1 in ARMS cells resulted in growth and c-jun phosphorylation.

Conclusion: In summary, these data suggest a model whereby the effect of ILK as an oncogene or tumor suppressor is determined by JNK1. Finally, this data suggests that ILK kinase inhibition may be warranted in ARMS tumors, and may be contraindicated in ERMS.

\section{STIMULATION OF TOLL-LIKE RECEPTOR 2 BY PLASMODIUM FALCIPARUM GLYCOSYLPHOSPHATIDYLINOSITOLS ENHANCES MACROPHAGE INTERNALIZATION OF PARASITIZED AND UNINFECTED ERYTHROCYTES}

\author{
Laura Erdman and Kevin C. Kain \\ McLaughlin-Rotman Centre for Global Health, University of
} Toronto, Toronto, Canada

Background: Toll-like receptors (TLRs) are highly conserved innate sensing receptors that activate host defenses upon detection of microbial products. Plasmodium falciparum glycosylphosphatidylinositols ( $P f \mathrm{GPI}$ ) have been shown to stimulate macrophage cytokine production via TLR2. In addition to their role in inflammation, TLRs may also regulate phagocytosis. $P$. falciparum parasitized erythrocytes (PEs) can be non-opsonically internalized by macrophages in a process predominantly mediated by scavenger receptor CD36. Moreover, uninfected erythrocytes (UEs) are rendered susceptible to macrophage clearance during malaria infection due to surface modifications, and this is believed to contribute to the pathogenesis of severe malarial anemia. We hypothesized that stimulation of macrophage TLR2 by PfGPI would enhance innate clearance of PEs as well as malaria-exposed UEs.

Methods: Primary human and murine macrophages were prestimulated with PfGPI or a synthetic TLR2 agonist (FSL-1) and phagocytosis assays were performed using $P$. falciparum PEs, EBABs (PE model consisting of anti-CD36 antibodies conjugated to human erythrocytes), IgG-opsonized PEs, and malariaexposed UEs.

Results: PfGPI and FSL-1 pre-stimulation significantly increased uptake of EBABs and $P$. falciparum PEs in a TLR2dependent manner. TLR2 stimulation modestly increased Fcmediated phagocytosis of IgG-opsonized PEs, and enhanced phagocytosis of UEs isolated from $P$. falciparum culture.

Conclusion: TLR2-mediated macrophage activation enhanced in vitro clearance of both PEs and malaria-exposed UEs. These data underscore the complexity of TLR involvement in malaria infection: TLR-enhanced phagocytosis may benefit infected individuals by decreasing parasite burden, but in other contexts may predispose to anemia by enhancing UE destruction. Therapeutic targeting of TLR pathways in malaria requires careful consideration. 


\section{MOTIVATION AND PLEASURE IN THE SCHIZOPHRENIA SPECTRUM - FINDINGS FROM THE PRODROME}

\author{
G. Foussias MSc $M D^{1,2}$, G. Remington MD PhD ${ }^{1,2}$, and $R$. \\ Mizrahi MD PhD \\ ${ }^{1}$ Centre for Addiction and Mental Health, and Department of \\ Psychiatry, University of Toronto, Toronto, Canada \\ ${ }^{2}$ Institute of Medical Science, Faculty of Medicine, University of \\ Toronto, Toronto, Canada
}

Background: Schizophrenia is a chronic and debilitating illness that affects approximately one percent of the population. The symptoms of schizophrenia are typically thought of in separate domains, including positive symptoms (hallucinations and delusions), negative symptoms (diminished emotional expression and amotivation), and cognitive deficits. Importantly, the negative symptoms have been consistently found to adversely influence functional outcomes, in particular due to marked amotivation. ${ }^{1}$ There have been suggestions that these individuals also experience deficits in the experience of pleasure, especially in their capacity to anticipate pleasure. ${ }^{2}$ However, such investigations have not included the examination of these symptoms in those in the prodromal phase of this illness, a time that holds promise for early intervention and altering the course of schizophrenia. ${ }^{3}$

Methods: In an effort to examine deficits in motivation and pleasure in the prodromal phase of schizophrenia, we have used an experience sampling method to assess "in the moment" motivation and pleasure in individuals at high risk of developing schizophrenia and healthy controls. Subjects completed baseline assessments including evaluation of their positive and negative symptoms. Subsequently, through the use of a personal digital assistant, subjects rated their motivation and experience of consummatory and anticipatory pleasure in their daily lives, multiple times over the course of four days.

Results and Conclusions: Preliminary data will be presented, as well as the importance of these findings in the context of understanding the underlying pathobiology of this illness, and guiding our search for effective treatments to improve functional outcomes in schizophrenia.

\section{References:}

1. Sayers SL, Curran PJ, Mueser KT. Psychol Assessment 1996;8:269-80.

2. Gard DE, Kring AM, Gard GM, et al.. Schizophr Res

2007;93:253-60.

3. Addington J. J Psychiatry Neurosci 2003;28:93-7.

\section{DISTINCT ROLES FOR DISCOIDIN DOMAIN RECEPTOR 1 (DDR1) EXPRESSED ON BONE MARROW DERIVED CELLS AND VESSEL WALL CELLS DURING ATHEROGENESIS.}

\section{Franco C, Hou G, Bendeck MP.}

Department of Laboratory Medicine and Pathobiology, Faculty of Medicine, University of Toronto. Toronto, Ontario, Canada

We have recently described a critical role for the discoidin domain receptor 1 (DDR1) collagen receptor tyrosine kinase in the regulation of fibrosis and inflammation during atherosclerotic plaque development. DDR1 is expressed on both SMCs and macrophages; however the role of DDR1 expressed in these distinct cells during atherogenesis remains unresolved. In the current study, female $L d l r^{-/}$mice that were either $\mathrm{Ddr1^{+/+ }}$ or $\mathrm{Ddr1^{-/ }}$ were lethally irradiated and reconstituted with bone marrow from male $D d r 1^{+/+}$or $D d r 1^{-/-}$donors yielding three groups of chimeric mice: $D d r 1^{+/+\rightarrow+/+}$ (control); $D d r 1^{+/+\rightarrow-/-}$ (vessel wall deletion); and $\mathrm{Ddrl}^{-/ \rightarrow+/+}$ (bone marrow deletion). Chimeric mice were placed on an atherogenic diet for 12 weeks and had similar body weights, total leukocyte counts, levels of Sry chimerism, and fasting plasma triglycerides at sacrifice, although total cholesterol was increased by $42 \%$ in $D d r 1^{+/+\rightarrow-/}$ mice. Deletion of DDR1 in bone marrow derived cells $\left(D d r 1^{-/ \rightarrow+/+}\right)$ resulted in a $66 \%$ reduction in atherosclerotic lesion area in the descending aorta compared to $D d r 1^{+/+\rightarrow+/+}$ mice. Aortic sinus plaques from $D d r 1^{-/ \rightarrow+/+}$ mice were $36 \%$ smaller than $D d r 1^{+/+\rightarrow+/+}$ plaques but the proportion of plaque area occupied by cells and matrix was similar between groups. By contrast, deletion of DDR1 in vessel wall cells $\left(D d r 1^{+/+\rightarrow-/}\right)$ resulted in a $57 \%$ increase in atherosclerosis in the descending aorta. Furthermore, aortic sinus plaques from $D d r 1^{+/+} \rightarrow-/$ mice had markedly increased fibrillar collagen and elastin accumulation compared to $D d r 1^{+/+\rightarrow+/+}$ plaques resulting in a $156 \%$ increase in lesion area and reduced SMC and macrophage content. In conclusion, while DDR1 on bone marrow derived cells is required for plaque development, DDR1 expressed on vessel wall cells negatively regulates plaque matrix accumulation and results in the formation of larger lesions with altered cellular composition. Our data suggest a dual role for DDR 1 in the regulation of atherogenesis and plaque matrix content. 


\section{EPIGENETIC ALTERATIONS ASSOCIATED WITH PREMATURE OVARIAN FAILURE}

\author{
Ghahremani Manda', Hannah Courtney $W^{2}$, Peneherrera \\ Maria $^{2}$, Bretherick Karla $L^{2}$, Fluker Margo $R^{1,3}$, Robinson \\ Wendy $P^{2}$ \\ ${ }^{1}$ Department of Obstetrics \& Gynecology, University of British \\ Columbia; ${ }^{2}$ Department of Medical Genetics, University of Brit- \\ ish Columbia; ${ }^{3}$ Genesis Fertility Centre, Vancouver
}

Background/Purpose: Premature ovarian failure (POF) affects $1 \%$ of women with a largely idiopathic and poorly understood etiology. The objective of this study was to identify specific epigenetic alterations by measuring DNA methylation of gene regulatory regions in women with POF vs. controls.

Methods: Blood samples were collected from idiopathic POF patients (Amenorrhea for at least 3 months and 2 serum FSH levels of $>40 \mathrm{mIU} / \mathrm{ml}$ obtained $>1$ month apart prior to age 40) and control women $(\mathrm{CW})$ (healthy pregnancy after age 37 with out a pregnancy loss). Genomic DNA was extracted from EDTA anticoagulated blood and bisulfite converted for analysis using the Illumina Golden Gate Methylation Panel which measures DNA methylation at $1506 \mathrm{CpG}$ sites in the promoter regions of 807 genes in $10 \mathrm{POF}$ and $12 \mathrm{CW}$. Candidate genes with altered epigenetic marks between $\mathrm{POF}$ and $\mathrm{CW}$ at a nominal $P$-value $<0.05$ were identified using a t-test comparison within the Illumina bead studio software. Genes of interest were further analyzed for quantitative methylation at specific $\mathrm{CpG}$ sites using pyrosequencing in $30 \mathrm{POF}$ and $30 \mathrm{CW}$.

Results: Comparison of DNA methylation profiles of our initial POF and CW groups identified several genes with statistically significant hyper- or hypo- methylation in the POF group $(P<0.05)$, including the Androgen Receptor (AR) promoter region, which was significantly hypermethylated. To further validate these results, DNA methylation of the AR gene promoter was quantified by pryosequencing in a larger group of POF and CW. Pyrosequencing further confirmed a significantly higher DNA methylation of the AR promoter region in POF vs. CW $(P=0.007)$.

Conclusions: This is a novel study identifying epigenetic alterations in POF. The hypermethylation of the AR gene in POF patients may cause decreased level of the AR in these women. This is especially interesting given a recent report of induced POF in AR deficient mice ${ }^{1}$. Specific epigenetic markers, as identified by DNA methylation array profiling in blood, may serve as useful biomarkers for POF and other fertility disorders. However, it will need to be determined if these methylation changes are present prior to diagnosis, or are a consequence of menopause itself.

\section{Reference:}

1. Hiroko S. et al. Premature ovarian failure in androgen receptor deficient mice. PNAS;103:224-9

\section{MUNC13 REGULATES SECRETION AT THE GOLGI IN A LOCALIZATION-DEPENDENT MANNER}

\author{
Neil M. Goldenberg* and Mel Silverman *广 \\ *Institute of Medical Science, †Department of Medicine, \\ University of Toronto, Ontario, Canada.
}

Background: Constitutive secretion is critical for the maintenance of eukaryotic cell structure and function. Our lab has shown that Rab34 is required for secretion at the Golgi ${ }^{1}$, and that the $\mathrm{C} 1$ domain-containing protein, Munc13, is an effector of Rab342. Current studies seek to elucidate potential roles for Munc13 in secretion at the Golgi.

Methods: Using a temperature-sensitive mutant of the Vesicular Stomatitis G-protein fused to GFP (VSVG-GFP) to monitor secretion, we examined the role of Munc13 in secretion in HeLa cells. Cells transfected with VSVG-GFP were treated with Munc13, a mutant lacking the $\mathrm{C} 1$ domain (C1-less), and the phorbol esters TPA and PDBu. The rate of VSVG-GFP secretion was monitored using surface labelling of plasmalemmal VSVGGFP and spinning disc confocal microscopy.

Results: TPA treatment resulted in an increase in the rate of VSVG-GFP appearance at the plasma membrane. Cotransfection of either Munc13 or C1-less alone also resulted in an increased rate of VSVG-GFP transport. Transfection of Munc13 plus TPA treatment resulted in a marked decrease in the rate of VSVG-GFP transport. Since TPA treatment relocalizes Munc13 to the plasma membrane, this result suggests that the availability of Munc13 in the cytosol is required for its effect on VSVG-GFP secretion.

Conclusions: Munc13 over-expression increases the rate of VSVG-GFP secretion to the plasma membrane. Sequestration of Munc13 at the plasma membrane with TPA abrogates this effect, and reduces the rate of VSVG-GFP secretion. We propose that Munc13 effects VSVG-GFP secretion via its interaction with Rab34 at the Golgi.

\section{References:}

1. Goldenberg, NM, S. Grinstein, M. Silverman. Golgi-bound Rab34 is a Novel Member of the Secretory Pathway. Mol Biol Cell. 18(12):4762-4771 (2007).

2. Speight, P, M. Silverman. Diacylglycerol-Activated Hmunc13 Serves as an Effector of the GTPase Rab34. Traffic. 6(10):858-865 (2005). 


\section{EFFECTS OF LOW NEIGHBOURHOOD SOCIOECONOMIC STATUS ON INFLAMMATION, OXIDATIVE STRESS, AND RISK OF MORTALITY IN PATIENTS WITH CORONARY ARTERY DISEASE}

\section{C.L. Heslop ${ }^{1,3}$, G. Miller ${ }^{2}$, J.S. Hill ${ }^{3}$}

${ }^{1} \mathrm{MD} / \mathrm{PhD}$ Program, University of British Columbia, Vancouver, BC. ${ }^{2}$ Department of Psychology, University of British Columbia, Vancouver, BC. ${ }^{3}$ Department of Pathology and Laboratory Medicine, The James Hogg iCAPTURE Centre for Cardiovascular and Pulmonary Research, University of British Columbia, Vancouver, $\mathrm{BC}$

Background: Socioeconomic status (SES) is an influential determinant of prognosis in coronary artery disease (CAD). Patient neighbourhood SES may contribute to CAD outcomes, beyond effects of personal SES.

Methods: Following 485 CAD patients for $>10$ years, we examine the effect of neighbourhood income, education, and unemployment on survival, and investigate relationships between SES and markers of inflammation and oxidative stress.

Results: SES was associated significantly with risk of mortality, however this relationship was not observed for cardiovascular death. Each one quintile decrease in income, education, and employment was associated with a $32 \%, 40 \%$ and $45 \%$ greater risk of non-cardiovascular mortality, respectively. Inflammatory and oxidative stress markers correlate with income, but do not diminish associations between neighbourhood SES and mortality.

Conclusions: Significant disparities in non-cardiovascular mortality related to neighbourhood SES were observed in this study, which argues for greater attention to socioeconomic factors in chronic disease prevention and health care delivery.

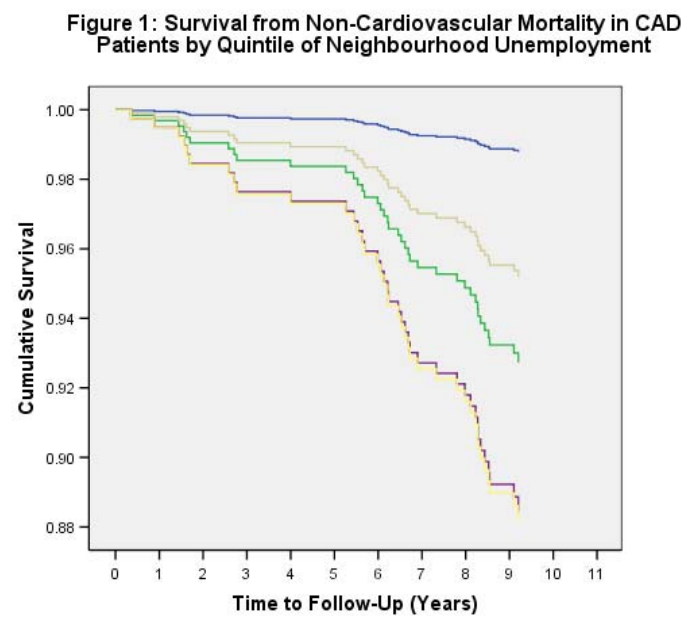

C.L.H. is supported by a Providence Health Research Institute \& Canadian Institutes of Health Research MD/PhD Studentship Award, and a Michael Smith Foundation for Health Research Trainee Award.

\section{TUMOUR PATHOLOGY PREDICTS MICROSATELLITE INSTABILITY IN A POPULATION-BASED SERIES OF COLORECTAL CANCER CASES}

\author{
A.J. Hyde ${ }^{1}$, D. Fontaine ${ }^{2}$, R.C. Green ${ }^{1}$, M. Simms ${ }^{1}$, P.S. Par- \\ frey $^{3}$, and H.B. Younghusband ${ }^{1}$ \\ Departments of ${ }^{1}$ Genetics, ${ }^{2}$ Pathology, and ${ }^{3}$ Clinical Epidemiol- \\ ogy, Faculty of Medicine, Memorial University of Newfoundland
}

Background: Lynch Syndrome is an autosomal dominant trait that accounts for approximately $3 \%$ of all cases of colorectal cancer (CRC). It is caused by mutations in DNA mismatch repair (MMR) genes, most commonly MLH1 or MSH2. These MMR defects cause high levels of microsatellite instability (MSI-H) in the tumours. MSI testing of all CRCs to identify potential Lynch Syndrome cases is not practical, so the Bethesda Guidelines, which use clinical and pathological features, were created to identify those tumours most likely to be MSI-H ${ }^{1}$. In 2007 Jenkins et. al. created MsPath, a tool based on the pathological features described in the rarely used $3^{\text {rd }}$ Bethesda criterion, to improve prediction of MSI-H tumours among CRC cases diagnosed before age 60 years ${ }^{2}$.

Methods: We collected a population-based cohort of 716 CRC cases diagnosed before age 75 years in Newfoundland. For each of these cases we collected family history, performed MSI analysis, and scored a number of pathological features for the purpose of evaluating the accuracy of the Bethesda Criteria and MsPath at predicting MSI-H tumours.

Results: Our work validates the MsPath tool in the Newfoundland population for the same age group used to create the tool. We found it identified MSI-H cases with a sensitivity of $95 \%$ and specificity of $35 \%$ in our population of CRC cases diagnosed before age 60 years $(n=290)$. We also tested this tool on our older population of CRC cases, diagnosed at ages 60 to 74 years $(n=426)$. We found it to be at least as predictive in this population, with a sensitivity of $95 \%$ and a specificity of $42 \%$. We then used our entire cohort $(\mathrm{N}=716)$ to compare MsPath with the other Bethesda criteria. Bethesda criteria 1,2, 4 and 5 together predicted MSI-H cases with a sensitivity of $67 \%$ and a specificity of $51 \%$. MsPath was better at identifying these cases, with a sensitivity of $95 \%$ and a specificity of $39 \%$.

Conclusions: We conclude that MsPath can be extended to include patients diagnosed with CRC before age 75 years. As well, we have found that MsPath is a better predictive tool than the Revised Bethesda Guidelines for identifying MSI-H cases within a population-based setting of colorectal cancer.

\section{References:}

1. Umar, A. et. al. J Natl Cancer Inst 2004;96:261-8

2. Jenkins, M.A. et. al. Gastroenterology 2007;133:48-56 


\section{PLASMACYTOID DENDRITIC CELLS FUNCTION IN HIV INFECTION.}

\section{Martin Hyrcza, Mario Ostrowski, Sandy Der.}

Dept. of Laboratory Medicine and Pathobiology, University of Toronto.

Plasmacytoid dendritic cells (pDCs) are innate immune cells able to produce large quantities of type I interferons (IFN) when activated. Human immunodeficiency virus (HIV)-infected patients show generalized immune dysfunction characterized in part by chronic interferon response. In this study we investigated the role of dendritic cells in activating and maintaining this response. Specifically we compared the IFN gene activity in pDCs in response to several viruses and TLR agonists. We hypothesized that 1) the pattern of IFN gene transcription would differ in pDCs treated with HIV than with other agents, and 2) that pDCs from patients from different stages of disease would respond differently to the stimulations. To test these hypotheses, we obtained pDCs from $15 \mathrm{HIV}$-infected and uninfected individuals and treated freshly isolated pDCs with either HIV (BAL strain), influenza virus (A/PR/8/34), Sendai virus (Cantell strain), TLR7 agonist (imiquimod), or TLR9 agonist (CpG-ODN) for 6h. Type I IFN gene transcription was monitored by real time qPCR for IFNA1, A2, A5, A6, A8, A17, B1, and E1, and cytokine levels were assayed by Cytometric Bead Arrays for TNF $\alpha$, IL6, IL8, IL10, IL1 $\beta$, and IL12p70. pDC function as determined by these two assays showed no difference between HIV-infected and uninfected patients or between patients with early or chronic infection. Specifically, HIV did not induce type I IFN gene expression, whereas influenza virus, Sendai virus and imiquimod did. Similarly, HIV failed to induce any cytokine release from $\mathrm{pDCs}$ in contrast to influenza virus, Sendai virus and imiquimod, which stimulated release of TNFa, IL6, or IL8. Together these results suggest that the reaction of pDCs to HIV virus is quantitatively different from the response to agents such as virus, Sendai virus, and imiquimod. In addition, pDCs from HIV-infected persons have responses similar to $\mathrm{pDCs}$ from uninfected donors, suggesting, that the DC function may not be affected by HIV infection.

\section{DEFICIENCY IN PROTEINASE-ACTIVATED RECEPTOR-2 (PAR2) ATTENUATES COLONIC INFLAMMATION IN MICE INDEPENDENT OF BONE MARROW DERIVED CELLS.}

\author{
Eric Hyun', Patricia Andrade-Gordon', and Nathalie \\ Vergnolle $^{1}$ \\ ${ }^{1}$ Department of Pharmacology and therapeutics, Faculty of medi- \\ cine, University of Calgary, ${ }^{2}$ Johnson Pharmaceutical Research \\ Institute
}

Background and aims: The role of $\mathrm{PAR}_{2}$ during intestinal inflammation is unclear, since its activation in the gut could lead to either pro- or anti-inflammatory properties. The aim of this study was to investigate the effects of $\mathrm{PAR}_{2}$ deficiency (using $\mathrm{PAR}_{2}$-deficient mice: $\mathrm{PAR}_{2}{ }^{-/}$) in an animal model of colitis and to investigate the role of $\mathrm{PAR}_{2}$ deficiency on bone marrowderived cells.

Methods: Colonic inflammation in $\mathrm{PAR}_{2}{ }^{+/+}$and $\mathrm{PAR}_{2}{ }^{-/-}$mice was induced by $2.5 \%$ dextran sodium sulfate (DSS). Chimeric mice $\left(\mathrm{PAR}_{2}{ }^{\mathrm{Ch}+/+}\right.$ or $\left.\mathrm{PAR}_{2}{ }^{\mathrm{Ch}-/}\right)$ injected with bone marrow cells (BMC) from either $\mathrm{PAR}_{2}{ }^{+/+}$or $\mathrm{PAR}_{2}{ }^{-/-}$mice, were also given DSS.

Results: $\mathrm{PAR}_{2}{ }^{+/+}$mice treated with DSS showed a significant increase in leukocyte rolling/adherence, bowel thickness, myeloperoxidase (MPO) activity and macroscopic damage compared to $\mathrm{PAR}_{2}{ }^{-/}$. $\mathrm{PAR}_{2}{ }^{\mathrm{Ch}-/}$ mice, regardless of the source of bone marrow cells injected, showed significantly reduced inflammatory parameters, compared to $\mathrm{PAR}_{2} \mathrm{Ch}+/+$ injected with $\mathrm{PAR}_{2}{ }^{+/+}$bone marrow. A similar degree of DSS-induced inflammation was observed between chimeric $\mathrm{PAR}_{2}{ }^{\mathrm{Ch}+/+}$ mice injected with either $\mathrm{PAR}_{2}{ }^{-/}$or $\mathrm{PAR}_{2}{ }^{+/+}$bone marrow cells.

Conclusions: Since DSS inflammation was reduced in $\mathrm{PAR}_{2}{ }^{\text {ch- } /-}$ compared with $\mathrm{PAR}_{2}{ }^{\mathrm{ch}+/+}$ mice, irrespective of the source of donor bone marrow cells, we conclude that $\mathrm{PAR}_{2}$ expression on recipient tissues rather than on donor bone marrow cells plays a key role in the development and maintenance of DSS-induced colitis. $\mathrm{PAR}_{2}$ thus appears as an important mediator of colonic inflammation and represents a potential target for the treatment of inflammatory bowel diseases.

Supported by CIHR and the Crohn's and Colitis Foundation of Canada 


\section{COMMITTED ADIPOGENIC PROGENITORS ARE RESPONSIBLE FOR NOVEL ADIPOCYTE GENERATION IN RESPONSE TO DIETARY STIMULI}

\author{
Aaron Joe ${ }^{1,2}$, Lin $\mathrm{Yi}^{2}$, A. Wayne Vogl ${ }^{3}$ and Fabio Rossi ${ }^{2}$ \\ ${ }^{1} \mathrm{MD} / \mathrm{PhD}$ Program, ${ }^{2}$ The Biomedical Research Centre, ${ }^{3}$ Depart- \\ ment of Cellular and Physiological Science, University of British \\ Columbia, Vancouver BC, V6T 1Z3
}

During adult life white fat depots can expand via hyperplasia and adipocyte infiltration can appear in aging or disease of nonadipose tissues. These observations suggest that stem/progenitor cells exist that can respond to a variety of stimuli to generate new adipocytes. We used a combination of surface markers to prospectively identify a population of cells containing all adipogenic colony-forming potential in skeletal muscle, subcutaneous and perigonadal adipose depots (adipogenic progenitors, APs). We did not observe any generation of cartilage, bone or muscle from AP cultures, suggesting that APs are committed to the adipocyte lineage. Confocal- and immunoelectron- microscopy analysis on tissues from BrdU-treated high fat diet-fed animals confirms the production of new adipocytes during weight gain. APs appear to participate in this response in a depot- specific manner. Furthermore, we observed a significant correlation between the size of the fat pad and frequency of actively cycling AP cells. Taken together, we show that we have identified an abundant population of lineage-restricted adipogenic progenitors. Importantly, our data suggests that during weight gain, new adipose generation relies on proliferation of these cells.

AJ is supported by a Vancouver Coastal Health -CIHR-UBC MD/PhD Studentship Award.

\section{LACK OF CELLULAR PRION PROTEIN UNMASKS NMDA NR2D SUBUNIT RECEPTOR FUNCTION WITH CONSEQUENCES TOWARD SYNAPTIC TRANSMISSION AND EXCITOTOXICITY}

Khosravani H, Zhang Y, Tsutsui S, Hameed S, Altier C, Hamid J, Chen L, Villemaire M, Ali Z, Jirik FR, Zamponi GW.

Department of Physiology and Biophysics, Hotchkiss Brain Institute, University of Calgary, Calgary T2N4N1, Canada.

Background: The physiological functions of endogenous cellular prion protein (PrPC) is incompletely understood. Previously, it has been shown that PrP-null mice exhibit reduced long-term (synaptic) potentiation and greater susceptibility to seizure mortality in several in vivo models of epilepsy. In addition, PrP-null neurons in culture exhibit greater excitotoxic cell death in response to kainic acid exposure, and in several models of oxidative stress. Although PrP seems to play a protective role against various forms of cellular insults, the precise mechanism of such action is unknown.

Methods: We investigated the synaptic properties of WT and PrPnull mice using cultured neurons and also brain slices from adult mice. Synaptic activity was assessed using whole-cell voltage clamp. We recorded spontaneous and evoked synaptic potentials. Extracellular field recordings of brain slices were also performed. Pharmacological agents were used to isolate all components of glutamatergic and $\operatorname{GABA}(\mathrm{A})$ mediated synaptic transmission. In addition, we assessed the effect of NMDA excitotoxicity in WT and PrP-null neurons using in vitro and in vivo experiments. We also used immunostaining, coimmunoprecipitation, and protein expression studies to quantify the relation between NMDA subtype expression and localization relative to native PrP.

Results: Recordings in the CA1 layer of adult hippocampal slices showed that PrP-null mice exhibit a reduced threshold to evoked responses, exhibited basal hyperexcitability, and in a model of zero-Mg2+ seizures also showed lower seizure threshold. No differences were observed in paired-pulse facilitation relative to WT animals. Recordings from mature hippocampal cultures showed slightly altered AMPA and GABAA miniature synaptic currents. NMDA mEPSCs were observed to be increased in amplitude and significantly prolonged in decay time. NMDA-evoked currents also exhibited markedly prolonged deactivation kinetics. This effect on evoked NMDA currents was reproduced in WT neurons by independent PrP-RNAi, NR2D-RNAi transfection, and eliminated by PrPC transfection into PrP-null neurons. In addition, PrP coimmunoprecipitated with NR2D and not NR2B NMDA receptor subunits. In vitro and in vivo experiments utilizing transient exposure to NMDA showed greater cell death in PrP-null neurons, which was significantly reduced by application of an NMDA receptor antagonist.

Conclusions: These data suggest that enhanced NMDA activity is present in PrP-null neurons. Consistent with this finding, in vitro and in vivo excitotoxicity assays demonstrated increased neuronal cell death in PrP-null cultures and animals upon transient exposure to NMDA. This was confirmed at the level of synaptic currents showing prolonged receptor deactivation kinetics that were most consistent with functional activation of NR2D NMDA receptor subunits. Enhanced NMDA receptor function was paralleled by increased excitotoxicity in PrP-null mice. Our findings demonstrate a novel functional role for PrP as a modulator of synaptic NMDA currents and attributes a neuroprotective function to PrP. 


\section{COPY NUMBER VARIATION IN METABOLIC SYNDROME \\ Lanktree M, Hegele RA}

Vascular Biology, Robarts Research Institute, Schulich School of Medicine \& Dentistry, University of Western Ontario

Metabolic syndrome (MetS) is defined by the presence of abdominal obesity, hypertension, dysglycemia, and dyslipidemia. Many mutations have been discovered that cause rare monogenic components of metabolic syndrome, and association studies have linked common variants with increased risk of MetS and its components. Despite successes in identifying genetic contributors to metabolic syndrome, unexplained heritability exists and copy number variation $(\mathrm{CNV})$ could be responsible for a portion of this variation. As observed with single nucleotide changes, it is likely that both rare and common CNVs will contribute to MetS disease susceptibility. Recent efforts to map CNVs in control populations have given insight into their size, frequency and distribution. However, despite being observed in controls, the reported CNVs could still modulate susceptibility for late-onset complex traits or produce subtle metabolic phenotypes. Here we examine the overlap between CNVs found in control datasets and genes with functional hypotheses or evidence of previous association to MetS. Secondly, we present the results and methodology of a search for a rare CNV in a high-penetrance Mendelian disorder, namely familial partial lipodystrophy. As methods to identify CNVs increase in precision and accuracy, the prospect of identifying their role in both rare Mendelian and common complex diseases is exciting.

\section{THE ROLE OF THE SRC-LIKE ADAPTOR PROTEINS, SLAP AND SLAP2, IN FLT3 RECEPTOR TYROSINE KINASE SIGNALING}

\section{Larissa Liontos and Jane McGlade.}

The Arthur and Sonia Labatt Brain Tumour Research Centre, The Hospital for Sick Children The University of Toronto, Department of Medical Biophysics

Introduction: The Src-like Adaptor Proteins, SLAP and SLAP2, are primarily expressed in hematopoietic cells and have an established role in the negative regulation of T-cell signaling. SLAP and SLAP2 act by recruiting the E3 ubiquitin ligase, c-Cbl, to components of the T-cell receptor leading to receptor ubiquitination and down-regulation. In addition to having a role in the negative regulation of $\mathrm{T}$ cell signaling, our lab has previously shown that SLAP and SLAP2 interact with the CSF-1 receptor, Fms, and interfere with CSF-1R function. The CSF-1 receptor is part of the type III family of receptor tyrosine kinases (RTKs) which includes the PDGF, c-Kit and Flt3 receptors. The Flt3 receptor plays an important role in both B-cell and Dendritic cell development and is commonly deregulated in acute myeloid leukemia (AML). The objective of this study is to determine whether SLAP and SLAP2 are involved in the negative regulation of Flt3 signaling.

Methods: To study Flt3 signaling in vivo, murine Dendritic cells (DCs) were utilized. We first analyzed the splenic DC populations in SLAP1/2-/- double knock-out mice and determined that there is a defect in the myeloid DC population $(\mathrm{CD} 11 \mathrm{~b} / \mathrm{CD} 11 \mathrm{c}+)$. To study receptor signaling, we generated DCs in vitro from the bone marrow of these mice using Flt3 ligand (Flt3L). SLAP1/ 2-/- mice produced less BM-DCs than WT mice and the functionality of these DCs is currently being investigated.

Results: SLAP and SLAP2 interact with the Flt3 receptor in vitro and bind to the receptor within the juxtamembrane region. BaF3/ Flt3 cells that stably express SLAP, SLAP2 and SH2 mutant forms of these proteins were generated. The expression of SLAP2 in these lines resulted in a change in the phosphorylation kinetics of the receptor. In addition, growth of SLAP2expressing cells in response to Flt3 ligand was enhanced in comparison to cells expressing a GFP vector alone.

Conclusions: SLAP and SLAP2 may be involved in Flt3 regulation and appear to play a role in Dendritic cell differenation, a process that is dependent on Flt3 signalling. 


\section{INVESTIGATING THE MOLECULAR AND DEVELOPMENTAL EFFECTS OF VARIOUS CULTURE REGIMES IN A MOUSE MODEL SYSTEM}

\author{
Brenna A. Market, Liyue Zhang, Lauren S. Magri, Michael C. \\ Golding, and Mellissa R.W. Mann \\ Children's Health Research Institute, Departments of Obstetrics \\ \& Gynaecology and Biochemistry, Schulich School of Medicine \\ and Dentistry, Children's Health Research Institute, The \\ University of Western Ontario, London, Ontario, Canada
}

Background/Purpose: Genomic imprinting is a specialized transcriptional mechanism that results in the unequal expression of alleles based on their parent-of-origin [1]. Many imprinted genes are critical for proper embryonic and fetal development [2] and disruption of genomic imprinting are associated with many development disorders [3]. Recently, increased frequencies of imprinting disorders have been correlated with the use of assisted reproductive technologies (ARTs)[2]. Rigorous and thorough testing of ARTs is required to determine their influence on genomic imprinting and development. I hypothesize that imprinting maintenance mechanisms are disrupted during early mouse development by the environmental insult of culture media used in human ARTs, and that loss of imprinting correlates with delayed embryonic development.

Methods: The specific aims of my project are to develop a method to evaluate the methylation and expression patterns of 4 known imprinted genes in individual blastocysts.

Results: We have successfully developed a novel method to evaluate both imprinted methylation and expression from a single mouse blastocyst. This method has been tested and results compared to methods used to evaluate imprinted methylation and expression separately; we have determined that results obtained with a combined protocol are equivalent to either alone. I will use this method to evaluate relationships between development rates in culture and genomic imprinting, as well as the effects of various culture media used for mouse and human embryo culture on genomic imprinting.

Conclusion: This analysis allow for a more comprehensive study of the effects of environmental insult on genomic imprinting and preimplantation embryo development.

\section{References:}

1. Reik W, Walter J. Genomic imprinting: parental influence on the genome. Nat Rev Genet 2001;2:21-32.

2. Rodenhiser D, Mann M. Epigenetics and human disease: translating basic biology into clinical applications. CMAJ. 2006;174:341-8.

3. Paoloni-Giacobino A. Epigenetics in reproductive medicine. Pediatr Res 2007;61:51R-57R.

\section{INTERACTION OF CD44 AND HYALURONAN IS THE DOMINANT MECHANISM FOR NEUTROPHIL ADHESION IN INFLAMED LIVER SINUSOIDS}

\author{
Braedon McDonald, Erin F. McAvoy, Florence Lam, Varinder \\ Gill, Paul Kubes \\ Immunology Research Group, Department of Physiology and \\ Biophysics, Institute of Infection, Immunity and Inflammation, \\ University of Calgary, Alberta, T2N 4N1, Canada
}

Background: Previous studies have been unable to identify adhesion molecules that mediate neutrophil recruitment within the liver sinusoids. We hypothesise that involved adhesion molecules may represent novel therapeutic targets for combating pathologic liver inflammation.

Methods: Candidate adhesion molecules were identified using a novel in vivo biopanning approach (dual radiolabelled antibody technique) to quantify endothelial expression levels within the liver compared to other organs. Spinning disk intravital microscopy demonstrated the localization of adhesion molecule expression within the liver microvasculature. Using knockout mice, bone marrow chimeric mice, and blocking antibodies, candidate adhesion molecules were systematically investigated for a role in neutrophil recruitment in the liver sinusoids of endotoxemic mice using intravital microscopy and in vitro flow chamber assays.

Results: Hyaluronan was identified as disproportionately expressed in the liver versus other organs, and hyaluronan expression was restricted to liver sinusoids. Blocking CD44hyaluronan interactions reduced neutrophil adhesion in the sinusoids of endotoxemic mice, but had no effect on neutrophil rolling or adhesion in post-sinusoidal venules. Neutrophil but not endothelial CD44 was required for adhesion in sinusoids. Surprisingly, neutrophil CD44 avidity for hyaluronan was not increased in endotoxemia. Instead, activation of CD44-hyaluronan engagement was the result of qualitative modification of hyaluronan by a dramatic induction of serum-derived hyaluronanassociated protein (SHAP) in sinusoids in response to lipopolysaccharide. Lipopolysaccharide-induced hepatic injury was significantly reduced by inhibiting CD44-hyaluronan interactions. Therapeutic administration of anti-CD44 antibody to endotoxemic mice rapidly detached adherent neutrophils and improved sinusoidal perfusion.

Conclusion: These findings reveal CD44 as a potential therapeutic target in systemic inflammatory responses involving liver. 


\section{CORTICAL AREA 5 IS NECESSARY FOR LONG-LASTING MEMORIES OF OBSTACLES ENCOUNTERED BY WALKING CATS}

\author{
David McVea ${ }^{1}$, PKG Pearson ${ }^{2}$ \\ ${ }^{1} \mathrm{MD} / \mathrm{PhD}$ Program, University of British Columbia, Vancouver \\ BC, V6T 1 Z3 \\ ${ }^{2}$ Centre for Neuroscience, University of Alberta, Edmonton, Al- \\ berta, T6G 2E8 \\ (Research conducted prior to UBC)
}

\begin{abstract}
Vision is used by walking animals to ensure stable foot placements and to avoid obstacles in their path. Visual input is not used directly when walking over normal terrain, however. Instead, walking animals look three or four steps ahead of their current position and use short-term memory to remember pertinent objects for use at the appropriate time. In cluttered terrain, humans may rely on increased visual input, guiding foot movement directly when needed. In quadrupeds, the hindlegs can never be guided by visual input directly, suggesting a specialized memory system might be used during walking in cluttered terrain. We have previously reported evidence in support of this hypothesis, showing that cats that have stepped over an object with their forelegs and stopped remember its position for much longer (for up to ten minutes) than if they are stopped prior to stepping over it.
\end{abstract}

In this presentation, I present research that tests the hypothesis that neural signals related to the movement of the forelegs are essential for the generation of this unique visual memory. By placing small lesions in cortical area 5, we disrupted the normal integration of sensory and motor signals in the parietal cortex. Cats with these lesions lost the long-lasting memory of straddled objects, and remembered these objects for no longer than if they had not stepped over them. The results support our hypothesis that a uniquely long-lasting memory system, initiated by neural signals related to foreleg stepping, is responsible for guiding the hindlegs of walking cats over obstacles.

Funding from Canadian Institutes of Health Research and Alberta Heritage Foundation for Medical Research gratefully acknowledged.

\section{GENOMIC AND TRANSCRIPTOME PROFILING OF EPITHELIAL OVARIAN CANCER}

Rebecca J.Z. Menzies ${ }^{1}$, Yury V. Bukhman', Nancy F. $\mathrm{Ng}^{2}$, Patricia A. Shaw ${ }^{1,3}$, Tak W. Mak ${ }^{1,2}$

${ }^{1}$ University of Toronto, ${ }^{2}$ Campbell Family Institute for Breast Cancer Research, ${ }^{3}$ Department of Lab Medicine and Pathobiology

Background: Epithelial ovarian cancer is the leading cause of death by gynecological malignancy. Due to inadequate screening modalities, a lack of characteristic presenting symptoms, limited treatments and a poor understanding of the molecular underpinnings of the disease, only $25 \%$ of ovarian cancers are diagnosed at an early stage. Current 5-year survival rates range from $80 \%$, for disease diagnosed in Stage I to as low as $13 \%$ for Stage IV. Current screening for ovarian cancer involves measuring CA-125 levels. However, CA-125 testing has low sensitivity since it can be elevated in a variety of other gynecological diseases. Numerous studies have found molecular heterogeneity between the four histological subtypes of ovarian cancer (serous, endometrioid, clear cell and mucinous). However, treatments remain the same for all subtypes regardless of molecular heterogeneity. Thus, better treatment targets and biomarkers must be found for this disease.

Methods: In our study 300 ovarian tumors will be genomically profiled using the Affymetrix Genome-Wide SNP Array 6.0 to identify loci and genes implicated in ovarian cancer. To date, 51 ovarian tumors have been analyzed using the SNP array.

Results: Preliminary analysis of copy number variation in these tumors using Partek software has revealed a total of 978 loci. Known amplifications derived from the literature were seen at CCNE1 and ERBB2. Similarly, well known deletions of p53 and RB1 in ovarian cancer were detected. Novel amplified loci at $18 \mathrm{q} 11.2$ and $4 \mathrm{q} 33$ were also detected. Novel deletions were detected at $7 \mathrm{p} 13$ and $8 \mathrm{q} 22.2$.

Conclusion: Future work will include running the remaining 249 tumor samples on the SNP array and analyzing the complete dataset using Partek software. Future validation of identified genes in vitro and in vivo may provide insight and possible biomarkers that may be used clinically to benefit the ovarian cancer patient. 


\section{INVESTIGATING CRMP4 FUNCTION IN NERVE REGENERATION}

\author{
S. Ong Tone ${ }^{1}$, Y.Z. Alabed ${ }^{1}$, A. Di Polo ${ }^{2}$, A.E. Fournier ${ }^{1}$ \\ Department of Neurology and Neurosurgery, Montreal Neuro- \\ logical Institute, McGill University ${ }^{1}$ \\ Department of Pathology and Cell Biology, Université de Mont- \\ real $^{2}$.
}

Background: The failure of CNS neurons to spontaneously regenerate following injury can be partially attributed to the expression of neurite outgrowth inhibitory myelin associated inhibitors (MAIs). MAIs signal through a tripartite receptor complex to activate the cytosolic protein RhoA and influence cytoskeletal dynamics. RhoA antagonists promote neuronal survival and regeneration in animal models of nerve injury. However, RhoA's potential as a therapeutic target may be limited by its widespread roles in multiple cellular processes and cell types. In an attempt to discover more specific therapeutic targets to promote nerve regeneration, our lab identified the cytosolic phosphoprotein CRMP4b (Collapsin Response Mediator Protein $4 \mathrm{~b}$ ) as a protein that functionally interacts with RhoA to mediate neurite outgrowth inhibition. Blockade of the RhoA-CRMP4b interaction with a competitive peptide (C4RIP) attenuates myelin-dependent neurite outgrowth inhibition.

Methods: We are currently investigating the in vivo roles of CRMP4 in regeneration in an optic nerve injury model by developing a readily deliverable version of C4RIP.

Results: Preliminary results suggest that overexpression of C4RIP in retinal ganglion cells by adeno-associated virus does not promote regeneration. However, studies investigating the ability of C4RIP to promote nerve regeneration into the optic nerve following stimulation of neurons into an active growth state are currently in progress.

Conclusion: Elucidating the role of CRMP4 in nerve regeneration may provide insight into the molecular mechanisms following nervous system injury and lead to the development of more specific therapeutic interventions.

\section{Reference:}

Alabed YZ, Pool M, Ong Tone S, Fournier AE. Identification of CRMP4 as a convergent regulator of axon outgrowth inhibition. J Neurosci 2007;27:1702-11.

\section{GENDER DIFFERENCES IN BLEEDING PROBLEMS AND IMPLICATIONS FOR THE ASSESSMENT OF A BLEEDING DISORDER}

M Pai ${ }^{1}, Y$ Li $^{1}, S$ Whittaker $^{1}$, E Arnold', JL Seecharan', KA

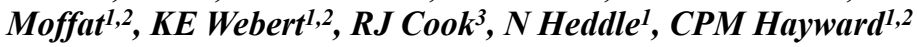
${ }^{1}$ McMaster University, ${ }^{2}$ Hamilton Regional Laboratory Medicine Program, ${ }^{3}$ University of Waterloo

Background: The value of gender-specific questions in assessing patients for bleeding problems is not well established, nor is the impact of bleeding on sexual and reproductive health.

Methods: We administered the CHAT (clinical history assessment tool) to 256 females and 66 males referred for bleeding problems, and 67 female and 32 male healthy controls. This detailed bleeding history questionnaire included questions about sexual health, blood in semen, and bleeding with circumcision, menses, pregnancies, and childbirth. A diagnosis for each patient was established by independent medical record review. The prevalence of symptoms among patients and controls was compared.

Results: $80 \%$ of patients had bleeding disorders (205 women, 54 men). Men with bleeding disorders did not have significantly increased intercourse-related bleeding, bleeding affecting their sex life, or gender-specific bleeding. Women with bleeding disorders had significantly increased intercourse-related bleeding and bleeding affecting their sex life. They also had significantly increased risks of prolonged menses, menses interfering with lifestyle, and menses requiring medical or surgical therapy (all $\mathrm{p}$ values $<0.001)$. Women with bleeding disorders were more concerned about pregnancy and delivery because of bleeding $(\mathrm{p}=0.0001$ ), and $38 \%$ had been told by a doctor not to become pregnant. Yet they did not have increased risks for pregnancy loss or bleeding during pregnancy ( $\mathrm{p}$ values $>0.1$ ) and had similar numbers of offspring as controls (mean 2.0 vs. 1.7).

Conclusion: Gender has an important impact on the manifestations, diagnosis and management of common bleeding disorders. Detailed questions about gender-specific bleeding are useful in assessing women with possible bleeding disorders, as they are at greater risk for bleeding that negatively impacts their sexual and reproductive health. 


\section{ETHICAL ANALYSIS IN PUBLIC HEALTH PRACTICE: A MULTI-SECTORAL MIXED- METHODS STUDY}

\section{Pakes B. Upshur REG.}

Joint Centre for Bioethics, University of Toronto

Rationale: All decisions in public health practice involve implicit value judgements, and many involve explicit reference to ethical principles. Despite the increased awareness, interest and literature in Public Health Ethics in the past decade, there remains little understanding of what public health practitioners or trainees mean by ethics, what meta-ethical foundations shape their approach to ethical dilemmas, and what prior training in ethics they have had or wish to have. This study aims to answer some of these questions and will serve as the basis for the development of resources to aid public health decision-making.

Method: Qualitative and quantitative data were collected from public health practitioners by means of paper and web-based surveys, as well as structured interviews. Data was coded and analysed using SPSS 15.

Results: 16/20 trainees, 70/150 Canadian practitioners, and 508/ 2058 American practitioners responded to the survey; 10 interviews were conducted. There was remarkable heterogeneity of responses regarding prioritization of values and meta-ethical justification of ethical norms. Respondents reported little training in ethics and considerable in enhancing their skills. Conflict between ethical imperatives and the law were a prominent feature of American, but not Canadian respondents.

Conclusions: Public Health practitioners hold a variety of disparate views regarding ethics in public health. These translate into different understandings of the goals and means of public health, with far reaching implications in all spheres of practice. A Public Health Ethical Reflection tool was developed to enhance ethical awareness in goal setting, planning and implementation of public health interventions.

\section{CONTRIBUTION OF DNA COPY-NUMBER VARIATION (CNV) TO CANCER SUSCEPTIBILITY AND LARGE-SCALE GENOME ALTERATIONS IN OSTEOSARCOMA (OS)}

Pasic $I^{1,2,3}$, Shlien $A^{1,4}$, Novokmet $A^{5}$, Zhang $C^{1}$, Tabori $U^{1,5,6}$, Pinto $D^{1,7}$, Scherer $S W^{1,7}$, Malkin $D^{1,2,4,5,6}$

Program in Genetics and Genome Biology ${ }^{1}$, Institute of Medical Science $^{2}, \mathrm{MD} / \mathrm{PhD}$ Program ${ }^{3}$, Department of Medical Biophysics $^{4}$, Division of Haematology/Oncology 5 , Paediatrics 6 , The Centre for Applied Genomics 7 , The Hospital for Sick Children, University of Toronto

Introduction: OS, a common Li-Fraumeni syndrome (LFS)associated neoplasm, is a common bone malignancy of children and adolescents. Sporadic OS is also characterized by young age of onset and high genomic instability, suggesting a genetic contribution to disease. This study examined the contribution of novel DNA structural variation elements, CNVs, to OS susceptibility. Given our finding of excessive constitutional DNA CNV in LFS patients, which often coincide with cancer-related genes, we hypothesized that constitutional CNV may also provide clues about the aetiology of LFS-related sporadic neoplasms like OS.

Methods: CNV in blood DNA of 26 patients with sporadic OS was compared to that of 263 normal control samples from the International HapMap project, as well as 62 local controls. Analysis was performed on DNA hybridized to Affymetrix genome-wide human SNP array 6.0 by Partek Genomic Suite.

Results: There was no detectable difference in average number of CNVs, CNV length, and total structural variation (product of average CNV number and length) between individuals with OS and controls. While this data is preliminary (small sample size), it argues against the presence of constitutional genomic instability in individuals with sporadic OS.

Conclusion: We found that the majority of tumours from patients with sporadic OS show CN loss at chr3q13.31, raising the possibility that chr3q13.31 may represent a "driver" region in OS aetiology. In at least one OS tumour, which displays CN loss at chr3q13.31, we demonstrate decreased expression of a known tumour suppressor gene located at chr3q13.31. We are investigating the role of chr3q13.31 in development of OS. 


\section{EVALUATION OF A NOVEL EEG ANALYSIS METHOD WITH POTENTIAL DIAGNOSTIC APPLICATIONS}

\author{
Peters, SR., $\mathrm{Hu}, \mathrm{B}$. \\ Department of Clinical Neuroscience, Hotchkiss Brain Institute, \\ University of Calgary, Calgary, AB, Canada
}

Background: Cerebral cortex oscillations as recorded on electroencephalograms involve multiple frequency bands. Phase locking of oscillations of these different frequencies may provide a mechanism by which regions of the brain communicate efficiently. Differences in the character of such phase locking may potentially be a diagnostic tool to differentiate seizure types., as traditional analysis of clinical EEG recordings has seldom considered phase-clocking as a diagnostic indicator.

Recently, Canolty et $\mathrm{al}^{1}$ used a novel metric to quantify crossfrequency phase-amplitude coupling during both spontaneous and induced EEG activity. The technique holds advantages over traditional measures, including easy comparison across trials, robustness to amplitude variation, and simple quantification of preferred phase. Traditional analysis of clinical EEG recordings has seldom considered phase-clocking as a diagnostic indicator.

Methods: We adapted the metric of Canolty ${ }^{1}$ to perform better with highly rhythmic oscillations, such as those in seizures, by adding multi-segment reshuffling of phase traces. To validate our modified technique, we used artificial sinusoid traces with a known degree of coupling to test the response of our modified analysis method, and to derive empirically, appropriate values for important numerical parameters. Frequency and phase information was acquired with both the Hilbert and wavelet transforms, with similar qualitative results achieved with either.

Results: As an initial exploration of diagnostic potential, we applied our metric to field potential data obtained from an anaesthetized rat preparation. We compared the phase-amplitude coupling profiles of sleep oscillations with those of simulated absence seizures and showed consistent differences in the phase amplitude coupling profiles. The data suggest that such differences may be useful in evaluating human seizure data.

Conclusions: We conclude that our modified method of data analysis provides an effective approach for measuring normalized phase-amplitude coupling in field potential recordings. Future work will aim to evaluate the possible diagnostic uses of phase-amplitude coupling analysis with data from human seizure patients.

Reference: Canolty et al. Science 2006;313:1626.

Supported by CIHR, NSERC, and the Health Research Foundation.

\section{SPECIES-SPECIFIC SEQUENCE VARIATION OF PORCINE ISLET AMYLOID POLYPEPTIDE REDUCES ITS AMYLOIDOGENICITY AND MAY CONTRIBUTE TO IMPROVED FUNCTION AND SURVIVAL OF PIG ISLET XENOGRAFTS.}

Potter KJ $J^{1}$, Abedini $A^{2}$, Butterworth $S^{1}$, Driscoll $M^{2}$, Marek $P^{2}$, Baker $R^{1}$, Korbutt $G^{3}$, Fraser $P E^{4}$, , Raleigh $D^{2}$, Verchere $\mathrm{CB}^{1}$

${ }^{1}$ Child and Family Research Institute, University of British Columbia, Vancouver, BC

${ }^{2}$ Department of Chemistry, Stony Brook State University of New York, Stony Brook, NY

${ }^{3}$ Department of Surgery, University of Alberta, Edmonton, AB

${ }^{4}$ Department of Medical Biophysics, University of Toronto, Toronto, ON

Introduction: Long-term success of human islet transplants has been limited. Beta cell mass and function in transplanted islets progressively decline over time, resembling the progressive loss of insulin secretion in type 2 diabetes. Transplanted islets, like those in type 2 diabetes, are subject to apoptotic beta cell death and the formation of toxic aggregates of the beta cell peptide islet amyloid polypeptide (IAPP). Interestingly, porcine islets consistently maintain long-term normoglycemia when transplanted into immune-deficient, diabetic mice. We hypothesized that transplanted pig islets may not be subject to amyloid formation and amyloid-induced cell death, and that this may contribute to the success of porcine islet grafts.

Methods \& Results: Sequencing of porcine IAPP (pIAPP) from neonatal pig islet (NPI)-extracted RNA confirmed notable differences from human IAPP (hIAPP), including a proline substitution within the known amyloidogenic region and a glutamine-forlysine at the N-terminal cleavage site of porcine proIAPP. Fibril formation was assessed by electron miscroscopy (EM) and Thioflavin $\mathrm{T}$ fluorescence. While synthetic hIAPP fibrillized within minutes, pIAPP required 6 weeks to form fibrils detectable by EM. Small peptide fragments of pIAPP failed to aggregate. By Thioflavin T fluorescence assay, hIAPP $(40 \mu \mathrm{M})$ had an average lag time of $1 \mathrm{~h}$ and a 1,000-fold increase in fluorescence by $2 \mathrm{~h}$ while pIAPP showed little increase in fluorescence after $20 \mathrm{~h}$. Small peptide fragments of pIAPP also failed to aggregate. hIAPP was significantly more toxic to INS-1 cells than pIAPP $(20-200 \mu \mathrm{M})$, as assessed by TUNEL (16 h) and Alamar blue (24 h). We also failed to detect amyloid in any (0/9) NPI transplanted into streptozotocin diabetic murine recipients, while human islets had extensive amyloid deposition by 8 weeks post-transplant, associated with graft failure.

Conclusion: Porcine IAPP, unlike human IAPP, is only weakly amyloidogenic and cytotoxic. We speculate that decreased fibrillogenicity of porcine compared to human IAPP may underlie the apparent enhanced survival and function of pig islet xenografts.

KJP is supported by a Child \& Family - Rx\&D \& CIHR - UBC MD PhD Studentship and a Michael Smith Foundation for Health Research Junior Trainee Award. 


\section{USE OF AN ELECTRONIC DATA WAREHOUSE TO ENHANCE CARDIAC SURGICAL SITE SURVEILLANCE AT A LARGE CANADIAN CENTRE.}

Rose $G W^{1}$, Roth $V R^{1,2}$, Suh $K N^{1}$, Taljaard $M^{1,2}$, van Walraven $C^{1,2}$, Forster $A^{1,2}$

${ }^{1}$ The Ottawa Hospital/University of Ottawa, ${ }^{2}$ Ottawa Health Research Institute

Background/Purpose: Surgical site infection surveillance to determine incidence is a key infection control activity. Case detection is labour-intensive, therefore most infection control programs use manual or simple electronic mechanisms to "trigger" chart review. However, such "trigger" mechanisms are also labour-intensive, and often of poor specificity. Our objective is to develop a complex trigger mechanism using data from an electronic data warehouse, to improve specificity of surveillance of surgical site infection compared to current trigger mechanisms.

Methods: We will derive an electronic trigger tool for cardiac surgical site infection surveillance using a nested case-control design, among a cohort of all patients undergoing coronary artery bypass grafting, cardiac valve repair or replacement, or heart transplant at the University of Ottawa Heart Institute, from July 1 2004 to June 302007.

We will perform a systematic literature review to identify potential trigger factors to include in the model, then construct the trigger tool by backwards stepwise logistic regression. The bestfit model will be used to calculate the probability of surgical site infection. We will select the threshold probability to use in surveillance by visual inspection of receiver-operator-characteristic curves. The accuracy of this electronic trigger mechanism will be compared to pre-existing manual and simple electronic mechanisms using relative true positive ratios and relative false positive ratios.

Results/Conclusions: We have selected 200 cases of surgical site infection and 541 controls from among 3744 procedures performed during the study period. As of the date of this abstract we are still undertaking the systematic review.

\section{GAIT ABNORMALITIES AND SENSORY CHANGES ACCOMPANY NEURO- INFLAMMATION IN ANIMAL MODELS OF DISC HERNIATION RADICULOPATHY}

Mohammed F. Shamji ${ }^{1,2}$, Kyle D. Allen ${ }^{1}$, Samuel B. Adams ${ }^{3}$, Stephen So ${ }^{1}$, Liufang Jing ${ }^{1}$, Jun Chen ${ }^{1}$, William J. Richardson $^{3}$, Lori A. Setton 1,3

${ }^{1}$ Dept of Biomedical Engineering, Duke University; ${ }^{2}$ Div. of Neurosurgery, The Ottawa Hospital; ${ }^{3}$ Div. of Orthopedic Surgery, Duke University Medical Center

Objective: The goal of this study was to evaluate gait and behavioral changes in an animal model of disc-herniation induced radiculopathy. A second objective was to correlate these functional changes to evidence of neuroinflammation and autoreactive lymphocyte immune activation.

Methods: The animal model of radiculopathy involved harvesting autologous nucleus pulposus (NP) from a tail intervertebral disc, and then exposing the L5 dorsal root ganglion (DRG) by hemilaminectomy and partial facetectomy. Experimental animals $(n=16)$ received NP placement onto the DRG and control animals $(n=16)$ underwent exposure only. At weekly time points, animals were evaluated for mechanical allodynia by Von Frey testing and for gait symmetry by digitized video analysis. At sacrifice, serum was evaluated for inflammatory cytokine content. The L5 DRGs were then evaluated by immunohistochemistry for mediators of inflammation and immune activation. Statistical analyses were at the $\alpha=0.05$ level of significance, with Bonferroni corrections for multiple comparisons when appropriate.

Results: Sensory testing revealed persistent mechanical allodynia in rats subjected to NP stimulus compared with the sham surgery group (Von Frey testing, $p<0.01$ ). Gait analysis reflected the functional consequence of this altered sensation revealing marked asymmetry and a preference to place load on the contralateral limb (symmetry index, $\mathrm{p}<0.01$ ). Serum cytokine expression was equivalent between groups, reaffirming that the sensation and behavioral changes observed in these animals results primarily from local inflammatory changes. Immunohistochemical analysis of the sectioned DRGs after sacrifice revealed equivalent post-surgical inflammatory activation (IL23, $\mathrm{p}=$ 0.47 ), but substantial immune activation in the NP group (IL17, $\mathrm{p}$ $=0.01$ ).

Conclusion: This model of radiculopathy provides the first evidence of altered gait symmetry and locomotor ability in animals subjected to non-compressive placement of NP tissue. Systemic inflammation was absent as expected, but mechanical allodynia, local inflammation, and autoreactive immune activation were observed. Future work involves therapeutic interventions targeting these changes to rescue animals from the phenotype of inflammatory radiculopathy. 


\section{THE ASSOCIATION OF LOBULAR BREAST CANCER WITH GERMLINE MUTATIONS OF CDH1}

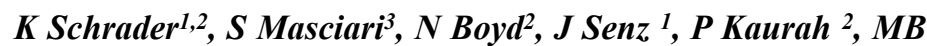
Terry $^{4}$, E John ${ }^{4}, I L$ Andrulis ${ }^{4}, J K^{2}$ night ${ }^{4}$, FP O'Malley ${ }^{4}, M$ Daly $^{4}$, P Bender ${ }^{4}$, MC Southey ${ }^{4,5}$, JL Hopper ${ }^{4,5,}$ J Garber ${ }^{3}$, DG Huntsman ${ }^{1,2}, \mathrm{kConFab}$.

${ }^{1}$ Department of Pathology and Laboratory Medicine, UBC, Vancouver, BC;

${ }^{2}$ Hereditary Cancer Program, BCCA, Vancouver, BC;

${ }^{3}$ Department of Medical Oncology, DFCI, Boston, MA;

${ }^{4}$ Breast Cancer Family Registry (B-CFR);

${ }^{5}$ University of Melbourne, Melbourne, Vic.

Background: $C D H 1$ encodes the cell-cell adhesion molecule, Ecadherin, for which loss of expression facilitates the infiltrative and metastatic potential of cancers. Germline mutations in $\mathrm{CDH} 1$ are associated with hereditary diffuse gastric cancer (HDGC), and in this setting female carriers have been estimated to have a $39-50 \%$ risk of lobular breast cancer (LBC) by age 80 years.

Aim: To determine the frequency of $\mathrm{CDH} 1$ germline mutations in individuals with early-onset LBC or those with LBC and a family history of multiple breast cancers but no gastric cancers.

Methods: Germline DNA analysis of $C D H 1$ in women with $\mathrm{LBC}$, for whom germline $B R C A 1$ and $B R C A 2$ mutations have been excluded, who have been (1) diagnosed before the age of 45 years or (2) diagnosed at any age and have a family history of breast cancer.

Results: Analysis of 194 LBC cases has thus far revealed two novel missense mutations predicted to affect protein function. Functional assays to assess their pathogenicity along with germline analyses of the remaining 200 cases are currently underway. Several unreported silent changes have also been identified and will be measured in a case- control sample to assess whether they are associated with LBC risk.

Conclusion: Germline $\mathrm{CDH} 1$ mutations may cause a small proportion of familial and early onset LBC.

\section{THE DYNACTOME: INVESTIGATING DYNAMIC KINASE NETWORKS IN CELLULAR DECISION-MAKING}

Jonathan So, Kelly Elder, Anna Dai, Claus Jorgensen, Rune
Linding, Karen Colwill, Tony Pawson Samuel Lunenfeld Research Institute, Mt. Sinai Hospital, 600 University Avenue, Toronto, ON, Canada M5G 1X5

Networks of kinases play a role in the transmission and integration of signals from the membrane to the nucleus. We aim to elucidate kinase phosphorylation and interaction partners in these networks through the immuno-precipitation and mass spectrometric analysis of a representative set of 100 Flag-tagged kinases stably expressed in human colorectal cancer cells. The goal is to generate a comprehensive set of interactions and dynamic phosphorylation sites which correlate with cell phenotypes such as apoptosis and proliferation. The techniques of massspectrometry have allowed for the identification of proteins and their phosphorylation sites in complex samples. Various labeling methods such as iTRAQ has enabled the relative quantification of these sites as a function of time (White et al. PNAS, 2007). However, kinases usually work in the context of particular signaling stimuli. We aim to characterize the role of these overexpressed kinases in the context of Trail-induced apoptosis. This is particularly relevant to tumorigenesis in that many cancers are resistant to apoptosis and recombinant Trail therapies are currently undergoing clinical trials. We present assays to correlate the proliferative ability and sensitivity to apoptosis of various stable cell lines with kinase expression levels through flow cytometry. We also present efforts to trace downstream signaling through the monitoring of MAP kinase phosphorylation using a high-throughput bead array. 


\section{ROLE OF THE CYSTIC FIBROSIS TRANSMEMBRANE REGULATOR (CFTR) CHLORIDE CHANNEL IN MACROPHAGE LYSOSOME ACIDIFICATION.}

\author{
Benjamin E. Steinberg, Alexandre Brodovitch, Kassidy K. \\ Huynh and Sergio Grinstein \\ Programme in Cell Biology, Hospital for Sick Children, Toronto \\ ON
}

Background: Lysosome acidification is the result of proton pumping by the vacuolar-type ATPase (V-ATPase). Because the V-ATPase is electrogenic, a substantial lysosomal membrane potential can develop if left uncompensated by counterions. An increasing membrane potential will oppose further proton pumping, limiting the acidification. It has generally been assumed that a parallel anion influx accompanies proton pumping to enable acidification. Indeed, defective anion channel function in cystic fibrosis (CF) has been suggested as the mechanism underlying attenuated lysosomal acidification and impaired microbial killing in the lung (Di A, et al., 2006, Nature Cell Biol. 8, 933-944). Chronic lung inflammation and infection represent the major source of morbidity and mortality in CF, and understanding the mechanism underlying the disease will therefore have farreaching therapeutic implications. As such, it is important to accurately evaluate the reported role of CFTR in lysosome acidification.

Methods: To assess the individual contribution of counterions to acidification, we carried out cytosolic and lysosomal ion substitution experiments in intact cells while monitoring lysosomal $\mathrm{pH}$ by ratiometric imaging.

Results: Replacement of cytosolic $\mathrm{Cl}^{-}$with impermeant anions did not alter the rate or extent of proton pumping. In contrast, permeant luminal cations were required for normal acidification. Because cations are the main counterion for lysosomal proton uptake, defects in the lysosomal $\mathrm{pH}$ are not anticipated in $\mathrm{CF}$ cells. Accordingly, the lysosomes of CFTR-deficient alveolar macrophages were found to acidify normally.

Conclusion: We conclude that cations are the primary counterions responsible for lysosomal acidification and that defects in lysosomal anion conductance cannot explain the impaired microbicidal capacity of CF phagocytes.

\section{SHCA PHOSPHOTYROSINE DERIVED SIGNALING IS REQUIRED FOR THE MAINTENANCE OF CARDIAC FUNCTION}

\author{
Rachel Vanderlaan 1, 2, 3, Rod Hardy', Golam Kabir ${ }^{2}$, Peter \\ Backx 2,4, AJ Pawson ${ }^{1,3}$ \\ ${ }^{1}$ Samuel Lunenfeld Research Institute at Mount Sinai Hospital \\ 600 University Ave; ${ }^{2}$. Heart and Stroke/Richard Lewar Research \\ Centre of Excellence, 150 College St.; ${ }^{3}$ Department of Molecular \\ Genetics, University of Toronto; ${ }^{4}$ Department of Physiology, \\ University of Toronto
}

Background: ShcA, a scaffolding protein, generates signal specificity by docking to activated tyrosine kinases through distinct phosphotyrosine recognition motifs, while mediating signal complexity through formation of diverse downstream phosphotyrosine complexes. Mammalian ShcA encodes 3 isoforms having a modular architecture of a PTB domain and SH2 domain, separated by a $\mathrm{CH} 1$ region containing tyrosine phosphorylation sites important in Ras-MAPK activation.

Objective and Methods: ShcA has a necessary role in cardiovascular development ${ }^{1,2}$. However, the role of ShcA in the adult myocardium is largely unknown, also unclear, is how ShcA uses its signaling modules to mediate downstream signaling. To this end, cre/loxP technology was employed to generate a conditional ShcA allele series. The myocardial specific ShcA KO (ShcA $\mathrm{CKO}$ ) and myocardial restricted domain mutant KI mice were generated using cre expressed from the mlc2v locus ${ }^{3}$ coupled with the ShcA floxed allele and in combination with the individual ShcA domain mutant KI alleles².

Results: ShcA CKO mice develop a dilated cardiomyopathy phenotype by 3 months of life, typified by depressed cardiac function and enlarged chamber dimensions. Isolated cardiomyocytes from ShcA CKO mice have preserved contractility indicating an uncoupling between global heart function and single myocyte contractile mechanics. Force-length experiments suggest that the loss of shcA mediates the uncoupling through deregulation of extracellular matrix interactions. Subsequent, analysis of the ShcA myocardial restricted domain mutant KI mice suggests that ShcA requires PTB domain docking to upstream tyrosine kinases and subsequent phosphorylation of the $\mathrm{CH} 1$ tyrosines important for downstream signaling.

Conclusion: ShcA is required for proper maintenance of cardiac function possibly : regulatioof extracellular matrix interactions.

\section{References:}

1. Lai KV, Pawson AJ. The ShcA phosphotyrosine docking protein sensitizes cardiovascular signaling in the mouse embryo. Genes and Dev 2000;14:1132-45.

2. Hardy WR. et al. Combinatorial ShcA docking interactions support diversity in tissue morphogenesis. Science 2007;317:251-6.

3. Minamisawa, s. et al. A post-transcriptional compensatory pathway in heterozygous ventricular myosin light chain 2deficient mice results in lack of gene dosage effect during normal cardiac growth or hypertrophy. J Biol Chem 1999;274:10066-70. 


\section{QUANTITATIVE EXAMINATION OF A NOVEL CLUSTERING METHOD USING MAGNETIC RESONANCE DIFFUSION TENSOR TRACTOGRAPHY}

Aristotle N Voineskos ${ }^{a, b}$, L J O'Donnell', NJ Lobaugh ${ }^{d}$, D Markant $^{e}$, M Niethammer ${ }^{e}$, BH Mulsant ${ }^{a}, B G$ Pollock $^{a}$, JL Kenne$d^{b}$, CF Westinf, ME Shenton ${ }^{*}$

${ }^{\mathrm{a} G e r i a t r i c ~ M e n t a l ~ H e a l t h ~ P r o g, ~ C A M H, ~ U n i v ~ o f ~ T o r o n t o ; ~}{ }^{\mathrm{b}} \mathrm{Dept}$ Neuroscience, CAMH, Univ of Toronto; ' $\mathrm{C}$, Clby Lab, Dept Neurosurgery, Brigham \& Women's Hospital, Harvard Univ; ${ }^{\mathrm{d} C o g n i t i v e ~ N e u r o l o g y, ~ S u n n y b r o o k ~ H o s p i t a l, ~ U n i v ~ o f ~ T o r o n t o ; ~}$ ePsychiatry Neuroimaging Lab, Dept of Psychiatry, Brigham \& Women's Hospital, Harvard University; f Lab for Math in Imaging, Dept of Radiology, Brigham \& Women's Hospital, Harvard Univ

Introduction: MR diffusion tensor imaging (DTI) is the most powerful and currently the only way to visualize the organization of white matter fiber tracts in vivo. As this is a relatively new imaging technique, new tools are developed for quantifying fiber tracts, and require evaluation. We examined scalar indices of the diffusion tensor with two different tractography methods. We compared a novel clustering approach with a multiple region of interest (MROI) approach in a healthy and disease (schizophrenia) population.

Methods: DTI images were acquired in 12 participants $(n=6$ patients with schizophrenia: $58 \pm 12$ years; $n=6$ controls: $57 \pm 21$ years) on a 1.5 Tesla GE system with diffusion gradients applied in 23 non-collinear directions, repeated three times. Tractography and fiber tract creation was performed using 3D Slicer software. Interrater reliability of the clustering approach and its similarity to the MROI method were evaluated.

Results: The clustering approach was reliable both quantitatively and spatially $(k>0.8$ for all tracts). There was high spatial (voxelbased) agreement between the clustering and MROI methods. Fractional anisotropy and trace values were highly correlated between the clustering and MROI methods ( $p<0.001$ for all tracts).

Discussion: Our clustering method has excellent interrater reliability and there is a high level of agreement between our clustering method and the MROI method, both quantitatively and spatially. The clustering method is less susceptible to user bias. Moreover, not limited by a priori predictions, our clustering method may be a more robust and efficient way to identify and measure fiber tracts of interest.

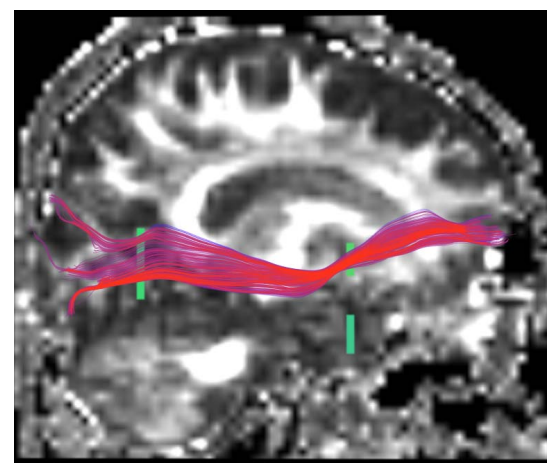

Right inferior occipitofrontal fasciculus showing MROI method and clustering method superimposed (high level of agreement in voxel space)

\section{ENDOTHELIAL NO-SYNTHASE GENE TRANSFER RESTORES REGENERATIVE CAPACITY OF ENDOTHELIAL PROGENITOR CELLS FROM PATIENTS WITH CORONARY ARTERY DISEASE}

\author{
Michael R. Ward ${ }^{1}$, Kathryn Isaac', Kathleen Thompson ${ }^{1}$, \\ Jonathan Vecchiarelli ${ }^{1}$, Michael J.B. Kutryk ${ }^{1}$ and Duncan J. \\ Stewart ${ }^{2}$ \\ ${ }^{1}$ St. Michael's Hospital, University of Toronto, Toronto, Ontario. \\ ${ }^{2}$ Ottawa Health Research Institute, Ottawa, Ontario.
}

Background: Endothelial progenitor cells (EPCs) from patients with coronary artery disease (CAD) or CAD risk factors exhibit greatly reduced regenerative capacity, which likely contributes to the relatively modest nature of the benefit seen in recent clinical trials of autologous cell therapy post myocardial infarction. We hypothesized that eNOS overexpression will improve the functional capacity of EPCs from these patients.

Methods and Results: EPCs were isolated from the peripheral blood of patients with high Framingham risk scores (FRS $>15 \%$ ) and were transduced using lentiviral vectors containing either eNOS or GFP (sham). We observed that eNOS-transduction significantly improved migration toward chemotactic factors (VEGF and SDF-1) compared to sham-transduced cells. EPCs were co-cultured with a mature endothelial cell (EC) line on Matrigel to measure their pro-angiogenic function in vitro. eNOStransduced EPCs induced longer angiogenic tubes with more branch points compared to sham-transduced cells, and exhibited higher association with EC tubes. We did not observe a significant difference in the adhesion of EPCs to an EC layer preactivated with TNF- $\alpha$, suggesting that the association to angiogenic tubes is likely through a different mechanism. In immunodeficient mice, eNOS-transduced EPCs resulted in significant improvement in ischemic hindlimb perfusion compared to shamtransduced cells. PCR arrays revealed changes in angiogenic and pro-survival gene expression in response to eNOS overexpression, providing preliminary insight into the mechanisms underlying its beneficial actions.

Conclusions: The present data show that reduced regenerative activity of EPCs isolated from CAD patients can be significantly improved by the overexpression of eNOS. The combination of cell and gene therapy may improve the efficacy of autologous cell therapies for cardiovascular disease. 


\section{HUMAN REGULATORY T CELLS PREVENT ISLET ALLOGRAFT REJECTION}

\author{
Douglas C. Wu ${ }^{1,2}$, Joanna Wieckiewicz', and Kathryn J. Wood ${ }^{1}$ \\ ${ }^{1}$ Nuffield Department of Surgery, University of Oxford \\ ${ }^{2} \mathrm{MD} / \mathrm{PhD}$ program, University of Alberta
}

Background: Type 1 diabetes mellitus represents a significant burden on global healthcare. Pancreatic islet transplantation offers an effective means of controlling the disease, but shortage of donor tissue, graft thrombosis, and immunological rejection after transplantation remain obstacles that need to be overcome. Our aim was to assess the ability of ex vivo expanded human regulatory $\mathrm{T}$ cells (Treg) in modulating the rejection response against a human islet allograft in a clinically relevant model of human pancreatic islet transplantation.

Methods: We studied the rejection response against allogeneic human islets in a cohort of 32 immunodeficient mice which had been reconstituted with a functional human immune system. Thirteen subjects were transplanted with human islets without further immunological modification; graft survival was compared with that of thirteen subjects treated additionally with human regulatory $\mathrm{T}$ cells. Six controls were given a human islet transplant, but not reconstituted with human immune cells to demonstrate the functionality of the islet graft in the absence of immunological rejection. Graft function was assessed with serial blood glucose measurements, immunohistochemistry, immunoflourescence, and flow cytometry.

Findings: Human islet allografts were rapidly rejected in subjects that did not receive Treg. With Treg treatment, however, human islet allograft rejection was prevented (median survival time (MST) of $>45$ days with Treg, as opposed to an MST of 23 days without Treg). Ex vivo expanded Treg homed to the lymphoid tissue draining the graft site where they suppressed the priming, activation, proliferation, and effector cytokine production of alloreactive $\mathrm{T}$ cells.

Interpretation: These findings in a clinically relevant model of human pancreatic islet transplantation demonstrate the ability of ex vivo expanded human Treg to attenuate acute islet allograft rejection, and provide further support for their use in cellular immunotherapy.

\section{IN UTERO GENE DELIVERY USING CHITOSAN-DNA NANOPARTICLES IN MICE}

\section{Yang PT, *Jia W, Skarsgard ED}

Division of Pediatric Surgery, *Division of Neurosurgery, Department of Surgery, University of British Columbia, Vancouver, British Columbia, Canada

Background: In utero gene therapy is a novel therapy for monogenic disorders of the fetus. Viral vector-mediated gene transfer risks endogenous viral recombination, and random transgene insertion causing insertional mutagenesis. Enhanced biosafety alternatives include non-viral vectors such as the complex polymer, chitosan. The purpose of this study was to evaluate chitosanmediated transfection in a murine model of fetal gene therapy.

\section{Methods:}

1. Chitosan colloidal suspensions were prepared, and particle size analysis in amniotic fluid (AF) was performed using a zetasizer.

2. Plasmid enhanced green fluorescent protein (eGFP)-chitosan constructs were prepared and protection from endogenous digestion in AF was analyzed by gel electrophoresis.

3. $0.25 \times 105$ HEK293T cells were transfected over 2 hours with $0.6 \mu \mathrm{g}$ of chitosan-peGFP in varying proportions of medium and AF. After $48 \mathrm{~h}$, cells were directly imaged by fluorescence microscopy for eGFP expression and fluorescence activated cell sorting (FACS) sorting was done to determine transfection efficiency. 4. Amniotic sacs of time-mated CD-1 mice were injected with 30 $\mu \mathrm{L}$ chitosan-peGFP (12.5 ug DNA) on G17. Following natural birth, pups were sacrificed and tissues were examined for eGFP DNA and mRNA by DNA PCR, RT-PCR, and fluorescence microscopy.

Results: Chitosan forms aggregates in AF, and although in vitro transfection efficiency is decreased by AF, eGFP-chitosan delivery into $\mathrm{AF}$ achieves transfection and transgene expression in lung and intestine of mice after birth.

Conclusions: In utero delivery of eGFP plasmid by chitosan results in postnatal gene expression, and shows promise for nonviral gene transfer in animal models of fetal gene therapy.

P.T.P.Y. is supported by a Child and Family-CIHR -UBC MD/ PhD Studentship Award. 


\section{REGULATION OF HUNTINGTIN PALMITOYLATION AND ITS ROLE IN HUNTINGTON DISEASE}

Fiona B.J. Young, 1,2,3, Deborah Yu Deng,3, Roshni R. Singaraja, 2,3, Michael R. Hayden ${ }^{2,3}$

${ }^{1} \mathrm{MD} / \mathrm{PhD}$ Program, University of British Columbia, Vancouver, $\mathrm{BC}$

${ }^{2}$ Centre for Molecular Medicine and Therapeutics, University of British Columbia, Vancouver, BC

${ }^{3}$ Child and Family Research Institute, University of British Columbia, Vancouver, BC

Huntington Disease (HD) is an inherited and ultimately fatal neurodegenerative disease demonstrating both neurological and psychiatric symptoms. The protein huntingtin (htt) undergoes many post-translational modifications, such as phosphorylation, palmitoylation, and proteolysis. Palmitoylation, the process by which a 16-carbon fatty acid forms a thioester bond with cysteine residues, is a reversible modification known to influence protein trafficking and function.

Huntingtin Interacting Protein 14 (HIP14) was identified as a major palmitoyl acyl transferase (PAT) that interacts robustly with wild-type htt, but has significantly reduced interaction with mutant polyglutamine expanded htt. HIP14 is a major PAT for $\mathrm{htt}$ and palmitoylation of mutant htt by HIP14 is significantly reduced. Downregulation of HIP14 by siRNA in vitro results in increased cell death in neurons, whereas co-transfection with htt and HIP14 results in enhanced palmitoylation and reduction in number of inclusions. Our laboratory has developed a HIP14 knockout mouse (HIP14-/-), which demonstrates many HD-like features similar to those seen in the YAC128 transgenic mouse model of HD. Notably, these mice demonstrate a much earlier and more severe phenotype as compared to the YAC128 mice, suggesting a critical role for HIP14 in HD pathogenesis.

The overarching goal of these studies is to explore the role of HIP14 and palmitoylation in the pathogenesis of HD using in vitro, in vivo, and ex vivo methods. A human Bacterial Artificial Chromosome (BAC) containing HIP14 was identified, prepared, and submitted for microinjection, and we have now generated HIP14-overexpressing transgenic mice. These mice are currently undergoing preliminary analyses, and a subset of founders will be selected to undergo further characterization. These human HIP14 BAC transgenic mice will represent the first mammalian model of PAT overexpression, and will significantly further our understanding of PAT activity in vivo.

The YAC transgenic model for HD, developed previously in our laboratory, recapitulates many aspects of $\mathrm{HD}$ as seen in humans. After preliminary characterization of the human HIP14 BAC transgenic mice, these mice will be crossed to the YAC model of HD. We anticipate that overexpression of HIP14 in vivo will delay and/or ameliorate the features of HD observed in the YAC128 mouse, providing validation of this pathway as a potential therapeutic target for HD.

F.B.J.Y. is supported by a Canadian Institutes of Health Research Walter and Jessie Boyd \& Charles Scriver and Child \& Family Research Institute -UBC MD/PhD Studentship Award. She also receives funding from the Michael Smith Foundation for Health Research as a Junior Trainee.

\section{RENAL OUTCOMES FOLLOWING HYDROXYETHYL STARCH RESUSCITATION: A META-ANALYSIS OF RANDOMISED TRIALS}

\author{
Ryan Zarychanski MD, Alexis F Turgeon MD MSc., Dean A \\ Fergusson MHA PhD, Deborah J Cook MD MSc, Paul Hébert \\ MD MSc, Sean M Bagshaw MD Msc, Danny Monsour, \\ Lauralyn McIntyre MD MHSc.
}

Objectives: To evaluate the impact of HES solutions on adverse renal outcomes and mortality in critically ill patients requiring acute volume resuscitation.

Design: Systematic review and meta-analysis of randomized controlled trials

Data Sources: We searched electronic databases from 1950 to 2007 (MEDLINE, EMBASE, the Cochrane Central Registry of Controlled Trials, and the SCOPUS database). Conference proceedings and grey literature sources were also searched from 2002-2007

Review Methods: We included all randomised controlled trials of patients requiring acute volume resuscitation who received HES compared to an alternative resuscitation fluid. No restrictions were considered regarding language or publication type. Data were independently extracted in duplicate.

Results: Of 2381 citations reviewed, we included 22 trials $(n=1866)$ in the analysis. Patients receiving HES were more likely to receive renal replacement therapy [odds ratio [OR] 1.91 (95\% confidence interval [CI] 1.22-2.99, $\left.\left.\mathrm{I}^{2} 10.5 \%\right)\right]$. This was also true for patients with severe sepsis or septic shock [OR 1.82 (95\% CI 1.27-2.62, $\left.\left.\mathrm{I}^{2} 0 \%\right)\right]$. In high quality trials, multicentre trials, and in reports indicating adequate allocation concealment, there was a trend toward increased risk of death associated with HES. Other adverse events were not systematically evaluated and were poorly reported.

Limitations: Considerable clinical and methodologic heterogeneity exists among these trials.

Conclusions: The use of HES for volume resuscitation in critically ill patients is associated with increased use of renal replacement therapy and may result in increased mortality. We caution against the routine use of HES for volume resuscitation in critically ill patients. 